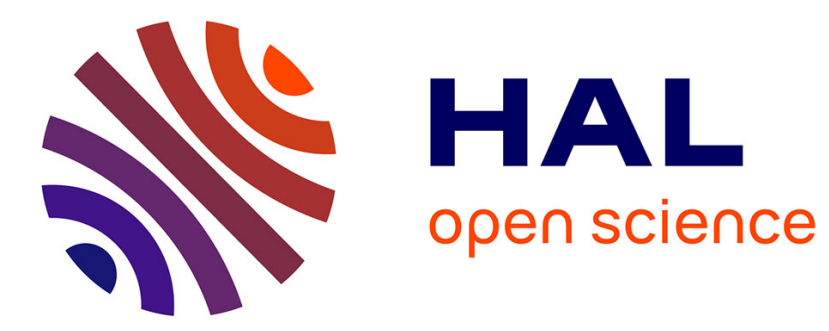

\title{
Numerical modeling of fluid flow and heat transfer in a narrow Taylor-Couette-Poiseuille system
}

\author{
Sébastien Poncet, Sofia Haddadi, Stéphane Viazzo
}

\section{To cite this version:}

Sébastien Poncet, Sofia Haddadi, Stéphane Viazzo. Numerical modeling of fluid flow and heat transfer in a narrow Taylor-Couette-Poiseuille system. International Journal of Heat and Fluid Flow, 2010, 32, pp.128-144. 10.1016/j.ijheatfluidflow.2010.08.003 . hal-00678877

\section{HAL Id: hal-00678877 https://hal.science/hal-00678877}

Submitted on 14 Mar 2012

HAL is a multi-disciplinary open access archive for the deposit and dissemination of scientific research documents, whether they are published or not. The documents may come from teaching and research institutions in France or abroad, or from public or private research centers.
L'archive ouverte pluridisciplinaire HAL, est destinée au dépôt et à la diffusion de documents scientifiques de niveau recherche, publiés ou non, émanant des établissements d'enseignement et de recherche français ou étrangers, des laboratoires publics ou privés. 


\title{
Numerical modeling of fluid flow and heat transfer in a narrow Taylor-Couette-Poiseuille system
}

\author{
Sébastien Poncet, ${ }^{*}$ Sofia Haddadi, ${ }^{\dagger}$ and Stéphane Viazzo ${ }^{\ddagger}$ \\ Laboratoire M2P2, UMR 6181 CNRS - Université d'Aix-Marseille - Ecole Centrale Marseille, \\ IMT la Jetée, 38 rue Joliot-Curie, 13451 Marseille (France)
}

(Dated: February 17, 2010)

We consider turbulent flows in a differentially heated Taylor-Couette system with an axial Poiseuille flow. The numerical approach is based on the Reynolds Stress Modeling (RSM) of Elena and Schiestel [1, 2] widely validated in various rotor-stator cavities with throughflow [3-5] and heat transfer [6]. To show the capability of the present code, our numerical predictions are compared very favorably to the velocity measurements of Escudier and Gouldson [7] in the isothermal case, for both the mean and turbulent fields. The RSM model improves, in particular, the predictions of the $k-\varepsilon$ model of Naser [8]. Then, the second order model is applied for a large range of rotational Reynolds $\left(3744 \leq R e_{i} \leq 37443\right)$ and Prandtl numbers $(0.01 \leq \operatorname{Pr} \leq 12)$, flow rate coefficient $\left(0 \leq C_{w} \leq 30000\right)$ in a very narrow cavity of radius ratio $s=R_{i} / R_{o}=0.961$ and aspect ratio $L=\left(R_{o}-R_{i}\right) / h=0.013$, where $R_{i}$ and $R_{o}$ are the radii of the inner and outer cylinders respectively and $h$ is the cavity height. Temperature gradients are imposed between the incoming fluid and the inner and outer cylinders. The mean hydrodynamic and thermal fields reveal three distinct regions across the radial gap with a central region of almost constant axial and tangential mean velocities and constant mean temperature. Turbulence, which is weakly anisotropic, is mainly concentrated in that region and vanishes towards the cylinders. The mean velocity distributions are not clearly affected by the rotational Reynolds number and the flow rate coefficient. The effects of the flow parameters on the thermal field are more noticeable and considered in details. Correlations for the averaged Nusselt numbers along both cylinders are finally provided according to the flow control parameters $R_{i}, C_{w}$ and $\operatorname{Pr}$.

Keywords: RANS modeling, Reynolds Stress Model, Taylor-Couette-Poiseuille flow, turbulence, heat transfer. 


\section{Nomenclature}

$A_{2}, A_{3} \quad$ Second and third invariants of the anisotropy tensor.

$C_{w} \quad$ Volume flow rate coefficient.

$h \quad$ Height of the cavity $(m)$.

$k \quad$ Turbulence kinetic energy $\left(\mathrm{m}^{2} / \mathrm{s}^{2}\right)$.

$L \quad$ Aspect ratio of the cavity.

$N \quad$ Rotation parameter.

$N u_{i}, N u_{o} \quad$ Local Nusselt numbers along the inner and outer cylinders respectively.

$\overline{N u_{i}}, \overline{N u_{o}} \quad$ Averaged Nusselt numbers for the inner and outer cylinders respectively.

$Q \quad$ Volume flow rate $\left(\mathrm{m}^{3} / \mathrm{s}\right)$.

$r, \theta, z \quad$ Cylindrical coordinates $(m)$.

$\operatorname{Pr} \quad$ Prandtl number.

$R e_{i} \quad$ Rotational Reynolds number based on the rotating velocity of the inner cylinder.

$R e_{t} \quad$ Turbulence Reynolds number.

$R_{i}, R_{o} \quad$ Radii of the inner and outer cylinders respectively $(m)$.

$R_{i j} \quad$ Reynolds stress tensor with $i, j=(r, \theta, z)\left(m^{2} / s^{2}\right)$.

$v_{r}^{\prime}, v_{\theta}^{\prime}, v_{z}^{\prime} \quad$ Fluctuating radial, tangential and axial velocity components $(\mathrm{m} / \mathrm{s})$.

$V_{r}, V_{\theta}, V_{z} \quad$ Mean radial, tangential and axial velocity components $(\mathrm{m} / \mathrm{s})$.

$\overline{V_{z}} \quad$ Averaged axial velocity imposed at the inlet $(\mathrm{m} / \mathrm{s})$.

$s \quad$ Radius ratio.

$T_{a} \quad$ Temperature of the incoming fluid $(K)$.

$T_{i}, T_{o} \quad$ Temperatures of the inner and outer cylinders respectively $(K)$.

$\delta \quad$ boundary layer thickness $(m)$.

$\Delta R \quad$ Hydraulic diameter $(m)$.

$\varepsilon \quad$ Dissipation rate of the turbulence kinetic energy $\left(\mathrm{m}^{2} / \mathrm{s}^{3}\right)$.

\footnotetext{
*Electronic address: poncet@l3m.univ-mrs.fr, Tel.33(4)91.11.85.23, Fax.33(4)91.11.85.02

${ }^{\dagger}$ Electronic address: sofia.haddadi@gmail.com

${ }^{\ddagger}$ Electronic address: stephane.viazzo@13m.univ-mrs.fr, Tel.33(4)91.11.85.49, Fax.33(4)91.11.85.02
} 
$\kappa_{i}, \kappa_{o} \quad$ Heating factors for the inner and outer cylinders respectively.

$\nu \quad$ Kinematic viscosity of the fluid $\left(\mathrm{m}^{2} / \mathrm{s}\right)$.

$\Omega \quad$ Rotation rate of the inner cylinder $(\mathrm{rad} / \mathrm{s})$.

$\Psi \quad$ Stream function

$\sigma$

Thermal diffusivity of the fluid $\left(\mathrm{m}^{2} / \mathrm{s}\right)$.

* denotes a normalized quantity.

a denotes a quantity for the incoming fluid.

i denotes a quantity on the inner cylinder.

o denotes a quantity on the outer cylinder. 


\section{INTRODUCTION}

The present investigation is concerned with the numerical modeling of fluid flow and heat transfer in a Taylor-Couette-Poiseuille system. The turbulent flow is confined between two differentially heated coaxial cylinders, with an inner rotating cylinder and a outer stationary one. An axial thoughflow of fresh fluid is also superimposed. This kind of Taylor-Couette flows with a superimposed Poiseuille flow is of great importance, since these flows have many applications in process engineering (dynamic membrane filtration, rheology, UV disinfection, pasteurization), geophysics (mantle convection) and also in the turbomachinery industry for bearings, asynchronous motor with axial ventilation [9], rotating heat exchangers and the drilling of oil wells among others. In the present work, the Taylor-Couette-Poiseuille system is a simple representation of the gap between the rotating and the stationary parts of an electrical motor contained in an air conditioning pack. A better knowledge of the convective heat transfer in the annular gap is required to optimize the perfomances of such a rotating machinery. Usually, the radial gap between the cylinders is quite weak (of the order of 1 $\mathrm{mm}$ ) and the rotation rate of the inner cylinder can reach more than $80000 \mathrm{rpm}$. The difficulty to perform accurate measurements in such closed clearances and especially in the very thin boundary layers along the cylinders has slowed down the development of specific turbulence models. It explains why relatively few works have been dedicated to such complex flows up to now.

The effect of an axial throughflow in a Taylor-Couette system, where only the inner cylinder is rotating, has been considered experimentally by Kaye and Elgar [10] in the isothermal case. Their results showed in particular the existence of four flow regimes depending on the Reynolds number based on the incoming flow velocity and the radial gap between the cylinders and on the Taylor number: laminar and turbulent flows, with or without Taylor vortices. Becker and Kaye [11] performed temperature measurements for a large range of rotation rate and superimposed flow rate. They studied the heat transfer in the gap between a heated inner rotating cylinder and a cooled outer stationary one. Compared to the isothermal case, they did not highlight the existence of Taylor vortices in the turbulent regime.

Most of the experimental works so far have been performed in Taylor-Couette systems characterized by a small aspect ratio $L=\left(R_{o}-R_{i}\right) / h$ and a large radius ratio $s=R_{i} / R_{o}$, with $R_{i}$ and $R_{o}$ the radii of the inner and outer cylinders respectively and $h$ the length of the cylinders. Aoki et al. [12] performed a combined theoretical and experimental investigation of turbulent Taylor-Couette flows without any Poiseuille flow. 
The most noticeable result is that the gap ratio $\left(R_{o}-R_{i}\right) / R_{i}$ in the range $[0.055-0.132]$ has only a small effect on the heat transfer for three different fluids: air, iso-buthyl alcohol and spindle oil. They provided also numerous correlations for the Nusselt number according to the Taylor and Prandtl numbers. Kuzay and Scott [13] studied experimentally the turbulent heat transfer in the gap between an inner rotating or non rotating insulated cylinder and an outer stationary and heated cylinder combined with an axial flow of air. They established correlations for the Nusselt numbers against a new physical parameter, called the rotation parameter $N$, which combines both the rotation and axial flow effects. This parameter is defined as the ratio between the rotating speed of the inner cylinder $\Omega R_{i}$ and the mean axial velocity of the incoming fluid $\overline{V_{z}}$. Lee and Minkowycz [14] highlighted experimentally the effects of the gap ratio $\left(R_{o}-R_{i}\right) / R_{i}$ in the range $[0.0083-0.051]$ and of grooved cylinders on the heat transfer. They showed in particular that the heat transfer process is enhanced for increasing values of the gap ratio. Escudier and Gouldson [7] performed velocity measurements by Laser Doppler Anemometry (LDA) in a cavity characterized by $L=0.0041$ and $s=0.506$ for various flow conditions (rotation rate $\Omega$ and flow rate $Q$ ) and different fluids including Newtonian and shear-thinning fluids. For the Newtonian fluid in the turbulent regime, the radial distribution of the axial velocity and the pressure drop are similar to the ones observed in pipe flows. The radial distribution of the tangential velocity reveals a flow structure divided into three regions: two very thin boundary layers, one on each cylinder, separated by a central core in near solid body rotation. The main effect of the superimposed axial throughflow is to reduce the tangential velocity in the core region. Nouri and Whitelaw [15] measured the three mean velocity components and the associated Reynolds stress tensor of the flow subjected to an axial superimposed throughflow in a concentric annulus $(L=0.0102, s=0.496)$ with or without rotation of the inner cylinder. Compared to the non-rotating case (for a given flow rate coefficient $\left.C_{w}=Q /\left(\nu R_{o}\right)=42306\right)$, the rotation of the inner cylinder at $\Omega=300 \mathrm{rpm}$ (rotational Reynolds number $\left.R e_{i}=\Omega R_{i}\left(R_{o}-R_{i}\right) / \nu=1616.6\right)$ does not affect the drag coefficient and the radial distribution of the mean axial velocity in the turbulent regime. It slightly enhances turbulence intensities especially close to the walls. For $C_{w}=125039$, there is absolutely no effect of the rotation of the inner shaft on both the mean axial flow and turbulence intensities. In a further paper, Nouri and Whitelaw [16] extended their work to the case of eccentric cylinder arrangements and proposed a review of previous works on Taylor-Couette flows including Newtonian and non-Newtonian fluids and rotating and non rotating flows. Bouafia et al. [17] 
performed extensive temperature measurements in the gap between a heated rotating inner cylinder and a cooled stationary outer one. An axial Poiseuille flow of air can be superimposed or not. The heat transfer is increased in the case of grooved cylinders compared to the basic case with smooth walls for an enclosed cavity. When an axial flow is imposed, the heat transfer along the rotating wall is increased in the smooth cylinder case. Numerous correlations for the Nusselt numbers along both surfaces are provided against a modified Taylor number and an effective Reynolds number for various flow conditions.

Naser [8] compared the predictions of a $k-\varepsilon$ model with the experimental data of Escudier and Gouldson [7] for the same flow conditions. For a turbulent Newtonian flow, the model showed large discrepancies for the mean velocity components. The profiles depend strongly on the axial position, which is not observed in the experiments [7]. Naser [8] attributed these deviations to the fact that the eddy viscosity concept, on which the model is developed, is incompatible with the simulated flow conditions. It can be attributed also to the fact that the $k-\varepsilon$ model is not sensitized to the implicit effects of rotation on turbulence. Char and Hsu [18] conducted numerical predictions using a modified version of the Launder-Sharma $k-\varepsilon$ model for turbulent mixed convection of air in a concentric horizontal rotating annulus. This model includes both the Yap correction and the Kato-Launder modification, which slightly improves the predictions compared to the classical Launder and Sharma model. The authors performed a parametric study of the fluid flow and heat transfer for various radius ratios $0.1 \leq s \leq 0.385$, Reynolds numbers $0 \leq R e_{i} \leq 10^{5}$ and Rayleigh numbers $10^{7} \leq R a \leq 10^{10}$. For this range of $R a$, they highlighted the three-dimensional structure of the flow with a two-cell structure in a $(r, \theta)$ plane for $R e$ up to $10^{4}$. Kuosa et al. [19] considered the cooling of high-speed electrical machines, where only the inner cylinder is rotating. They compared the predictions of three different models: an algebraic modeling, a low-Reynolds number $k-\varepsilon$ modeling and a $k-\omega$ SST model. The hydrodynamic and thermal fields are established for various rotation rates and mass flow rates. The three turbulence models underestimated the heat transfer coefficients along both cylinders. Moreover, the entrainment coefficient of the fluid was far from the theoretical value for a fully turbulent flow. These discrepancies were attributed by the authors to the boundary conditions imposed at the inlet and outlet sections and to the turbulence models used. Very recently, Giret [9] performed a combined experimental and numerical investigation of the convective heat transfers in the air gap of an alternator. Heat transfer measurements were compared to two-equation models contained within the commercial code ANSYS CFX for 
various flow conditions and different geometries for the inner rotating cylinder. The convective heat transfers were underestimated by the models on the rotor and overestimated on the stator. The experimental results were found to be almost the same when the inner cylinder is smooth or with four inter-polar gaps.

To our knowledge, Chung and Sung [20] were the first to perform Large Eddy Simulation (LES) in such complex configurations. They compared their numerical data to the experimental ones of Nouri and Whitelaw [15] for $s=0.5, C_{w}=20970$ and $R e_{i}=[954.5 ; 1909.1 ; 3818.1]$. They obtained a relatively close agreement for the mean velocity and the Reynolds stress tensor components. The mean tangential velocity was slightly overestimated in the main part of the radial gap between the cylinders. They focused their numerical investigation on the appearance of turbulent structures attributed to the destabilization of the flow along the inner rotating cylinder and giving rise to strong events (sweeps and ejections).

The purpose of this work is to predict the turbulent flow and heat transfert in the gap of an electrical machine (schematized here by a very narrow Taylor-Couette-Poiseuille system) for a wide range of operating conditions (see Table I). As there is absolutely no experimental or numerical evidence of three-dimensional structures embedded in such flows [11], two-dimensional calculations can be performed without any loss of information. The Reynolds Stress Model (RSM) of Elena and Schiestel [1, 2, 21], which has shown to offer the best trade-off between accuracy and calculation cost in various rotating flow arrangements [3-6, 22], has thus been chosen for this study. We propose here to extend, for the first time, the application of its well established turbulence model to real operating flow conditions in the rotor-stator gap of an electrical motor. It is also to get a better insight into the dynamics and the heat transfer process of the turbulent Taylor-Couette-Poiseuille flow in a very narrow cavity.

The paper is organized as follows: the geometrical configuration and the numerical modeling are described in Sections II and III respectively. Comparisons between the present calculations and the LDA measurements of Escudier and Gouldson [7] are performed in section IV to show the capability of the RSM model to predict such complex flows under isothermal conditions. The predictions of the RSM are extended to non-isothermal Taylor-Couette-Poiseuille flows in Sections V and VI. Section V is devoted to the detailed analyzis of the hydrodynamic and thermal fields for a given set of parameters $\left(R e_{i}=10216, C_{w}=14858, \operatorname{Pr}=0.7\right)$. The influence of the Reynolds and Prandtl numbers and of the flow rate are investigated in section VI. Finally some conclusions and closing remarks are provided in section VII. 


\section{GEOMETRICAL CONFIGURATION AND FLOW CONTROL PARAMETERS}

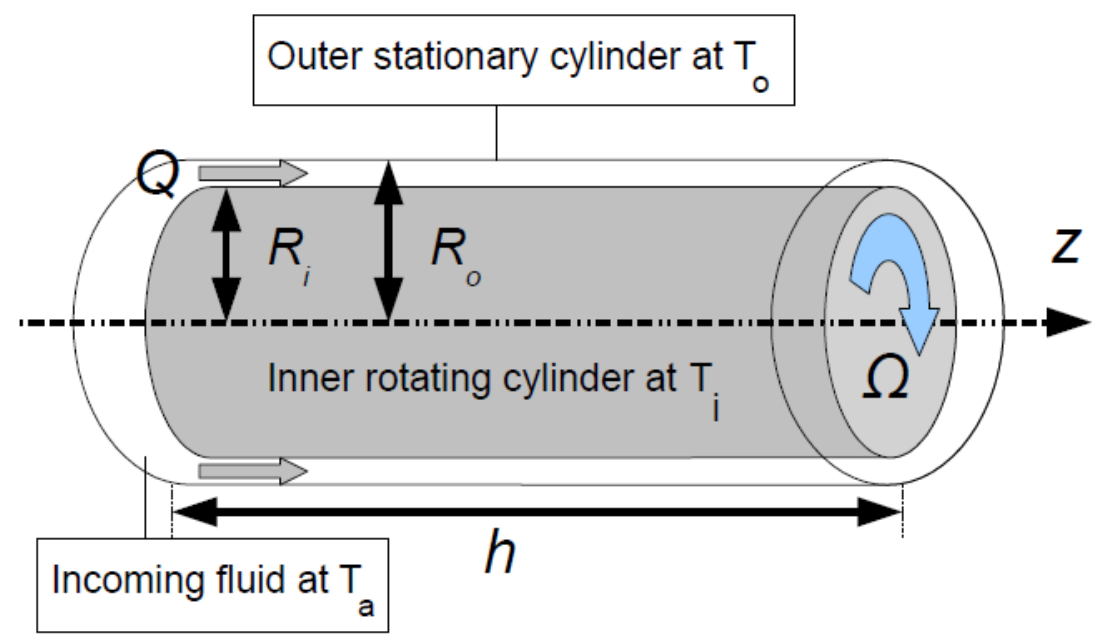

Figure 1: Schematic representation of the Taylor-Couette-Poiseuille configuration with relevant notations.

The cavity sketched in figure 1 is composed of two smooth concentric cylinders. The inner cylinder of radius $R_{i}$ is rotating at a given rotation rate $\Omega$, while the outer cylinder of radius $R_{o}$ is stationary. This configuration is known in the litterature as the Taylor-Couette problem. The height of the cavity is denoted $h$ in the following. An axial volume flow rate $Q$ can be superimposed at the cavity inlet.

The mean flow is mainly governed by four flow control parameters: the aspect ratio of the cavity $L$, its radius ratio $s$, the rotational Reynolds number $R e_{i}$ based on the rotating speed of the inner cylinder $\Omega R_{i}$ and half the hydraulic diameter $\Delta R=R_{o}-R_{i}$ and the flow rate coefficient $C_{w}$ defined as follows:

$$
\begin{gathered}
\mathrm{L}=\frac{R_{o}-R_{i}}{h} \quad s=\frac{R_{i}}{R_{o}} \\
R e_{i}=\frac{\Omega R_{i}\left(R_{o}-R_{i}\right)}{\nu} \quad C_{w}=\frac{Q}{\nu R_{o}}
\end{gathered}
$$

where $\nu$ is the fluid kinematic viscosity. Two different geometries will be considered in the following. Configuration 1 corresponds to the experiments performed by Escudier and Gouldson [7] under isothermal conditions (section IV) and Configuration 2 to another case including heat transfer effects (sections V and VI). The values of all parameters for these two configurations are summarized in Table I. The value $C_{w}=0$ corresponds 
to a closed cavity. The parameter $R e_{i}$ is also known as a Taylor number. The values of $R e_{i}$ considered here are much higher than the critical value $R e_{i}=210$ for the transition to turbulence found experimentally by Aoki et al. [12], which ensures that the flow is highly turbulent without Taylor vortices.

\begin{tabular}{c|c|c|c|c|c|c|c} 
& $L$ & $s$ & $R e_{i}$ & $C_{w}$ & $\operatorname{Pr}$ & $\kappa_{i}$ & $\kappa_{o}$ \\
\hline \hline configuration 1 & 0.0041 & 0.506 & 961.1 & $2839 ; 5914 ; 17742$ & - & - & - \\
\hline configuration 2 & 0.013 & 0.961 & {$[3744-37443]$} & {$[0-30000]$} & {$[0.01-12]$} & 0.117 & 0.245
\end{tabular}

Table I: Values of the flow control parameters for Configuration 1 corresponding to the experiments of Escudier and Gouldson [7] and Configuration 2.

Concerning the boundary conditions for the temperature field $T$ in Configuration 2, the fluid at the inlet enters the cavity at a constant temperature denoted $T_{a}$. The inner and outer cylinders are maintained at constant temperatures $T_{i}$ and $T_{o}$ respectively. Thus, the heat transfer is driven by three main parameters, two heating factors $\kappa_{i}$ and $\kappa_{o}$ for the inner and outer cylinders respectively and the Prandtl number $P r$ defined as follows:

$$
\kappa_{i}=\frac{T_{i}-T_{a}}{T_{a}}=0.117 \quad \kappa_{o}=\frac{T_{o}-T_{a}}{T_{a}}=0.245 \quad 0.01 \leq \operatorname{Pr}=\frac{\nu}{\sigma} \leq 12
$$

with $\sigma$ the thermal diffusivity of the fluid. The two heating factors $\kappa_{i}=0.117$ and $\kappa_{o}=0.245$, which are proportional to $G r / R e_{i}^{2}$, where $G r$ is the Grashof number based on the temperature $T_{a}$, are small enough to make the hypothesis of no density variation as the gravitational effects are small compared to the inertial effects $\left(G r<<R e_{i}^{2}\right)$.

The relevant parameters to study heat transfer are the local Nusselt numbers along the inner and outer cylinders defined as:

$$
\begin{aligned}
N u_{i}(z) & =\left.\frac{R_{o}-R_{i}}{T_{i}-T_{a}} \frac{\partial T}{\partial r}\right|_{i}(z) \\
N u_{o}(z) & =\left.\frac{R_{o}-R_{i}}{T_{o}-T_{a}} \frac{\partial T}{\partial r}\right|_{o}(z)
\end{aligned}
$$

We also define the averaged Nusselt numbers $\overline{N u_{i}}$ and $\overline{N u_{o}}$, which are the averaged values of the local Nusselt numbers along the heated surfaces, defined as follows: 


$$
\begin{aligned}
& \overline{N u_{i}}=\left.\frac{R_{o}-R_{i}}{h} \frac{1}{T_{i}-T_{a}} \int_{0}^{h} \frac{\partial T}{\partial r}\right|_{i}(z) d z \\
& \overline{N u_{o}}=\left.\frac{R_{o}-R_{i}}{h} \frac{1}{T_{o}-T_{a}} \int_{0}^{h} \frac{\partial T}{\partial r}\right|_{o}(z) d z
\end{aligned}
$$

\section{STATISTICAL MODELING}

The predictions of the present Reynolds Stress Model (RSM) developed by Elena and Schiestel [1, 2, 21] have already been validated in various rotating cavities including rotor-stator configurations with throughflow [3-6] and Von Kármán flows between counter-rotating impellers [22] for different geometries and a wide range of rotation rate, imposed throughflow and type of fluid. Elena and Schiestel [21] showed that this level of closure is adequate in such flow configurations, while the usual $k-\epsilon$ model, which is blind to any rotation effect presents serious deficiencies. The reader can refer to the previous works of Elena and Schiestel $[1,2,21]$ and Poncet et al. [3-6] for more details about the statistical modeling.

\section{A. The differential Reynolds Stress Model}

The flow studied here exhibits several complexities (high rotation rate, imposed throughflow, wall effects, strong curvature), which are a severe test for turbulence modeling methods. Our approach is based on onepoint statistical modeling using a low Reynolds number second-order full stress transport closure derived from the Launder and Tselepidakis [23] model and sensitized to rotation effects by Elena and Schiestel [1, 2]. It corresponds to the RSM3 model fully described in [1]. This approach allows for a detailed description of near-wall turbulence and is free from any eddy viscosity hypothesis. The general equation for the Reynolds stress tensor $R_{i j}$ can be written:

$$
\frac{d R_{i j}}{d t}=P_{i j}+D_{i j}+\Phi_{i j}-\epsilon_{i j}+T_{i j}
$$

where $P_{i j}, D_{i j}, \Phi_{i j}, \epsilon_{i j}$, and $T_{i j}$ respectively denote the production, diffusion, pressure-strain correlation, dissipation and extra terms. The diffusion term $D_{i j}$ is split into two parts: a turbulent diffusion $D_{i j}^{T}$, which is interpreted as the diffusion due to both velocity and pressure fluctuations [24] and a viscous diffusion 
$D_{i j}^{\nu}$, which cannot be neglected in the low Reynolds number region. In a classical way, the pressure-strain correlation term $\Phi_{i j}$ can be decomposed as below:

$$
\Phi_{i j}=\Phi_{i j}^{(1)}+\Phi_{i j}^{(2)}+\Phi_{i j}^{(w)}
$$

$\Phi_{i j}^{(1)}$ is interpreted as a slow nonlinear return to isotropy and is modeled as a quadratic development in the stress anisotropy tensor, with coefficients sensitized to the invariants of anisotropy. This term is damped near the wall. The linear rapid part $\Phi_{i j}^{(2)}$ includes cubic terms. A wall correction $\Phi_{i j}^{(w)}$ is applied to the linear part which is modeled using the Gibson and Launder hypothesis [25] with a strongly reduced numerical coefficient. However the widely adopted length scale $k^{3 / 2} \varepsilon^{-1}$ is replaced by the length scale of the fluctuations normal to the wall. The viscous dissipation tensor has been modeled in order to conform with the wall limits obtained from Taylor series expansions of the fluctuating velocities [26]. The extra term $T_{i j}$ accounts for implicit effects of the rotation on the turbulence field. Indeed, high speed rotation produces indirect effects on the turbulence field that are not modeled in usual closures, even in second order closures. These effects modify the structure of the turbulence eddies in a complex manner that can be evidenced in two-point statistics [27]. A practical extension for one-point closures, to approximate the effects, has been developed by Elena and Schiestel $[1,2]$. It consists in additionnal terms in the stress transport equations that act only when the flow is subjected to strong rotation. More precisely, the pressure-strain correlation is sensitized to the Reynolds and Cambon structure tensor. A spectral jamming term that enhances bidimensionality and the blocking effect of the spectral transfer are also included. These terms are fully explained and detailed analytically in $[1,3]$ and their influences will be discussed in the following.

For the thermal field, as we consider only relatively small temperature differences, density is not significantly affected, which allows to dissociate the dynamical effects from the heat transfer process. Poncet and Schiestel [6] obtained indeed very satisfactory results using this approach for temperature differences up to $75 \mathrm{~K}$ in similar geometries. Thus, the temperature equation writes:

$$
\frac{\partial T}{\partial t}+V_{j} T_{, j}=\sigma T_{, j j}-F_{j, j}^{t}
$$

where $F_{i}^{t}$ is the turbulent flux approximated by a gradient hypothesis with a tensorial diffusive coefficient: 


$$
F_{i}^{t}=-c_{t} \frac{k}{\varepsilon} R_{i j} T_{, j}
$$

with $c_{t}=c_{\mu} / P r_{t}=0.1, c_{\mu}=\nu_{t} \varepsilon / k^{2}=0.09$ a coefficient used to define the turbulent viscosity $\nu_{t}$ and $P r_{t}=0.9$ the turbulent Prandtl number. It is indeed a common feature for two-dimensional computations in rotating flows and more generally for near-wall turbulent flows to fix the value of the turbulent Prandtl number $\operatorname{Pr}_{t}$ to 0.9 (see the monographs of Launder et al. [28] and Schiestel [29]). The numerical work of Ong [30] and Iacovides and Chew [31] can also be cited.

The effects of the anisotropy of the turbulence field and the implicit effects of rotation are included in the term $k R_{i j} / \varepsilon($ Eq.8) for most of them.

\section{B. Numerical method}

The computational procedure is based on a finite volume method using staggered grids for mean velocity components with axisymmetry hypothesis in the mean and non staggered grids for the Reynolds stress tensor. The code is steady elliptic. The velocity-pressure coupling is solved using the SIMPLER algorithm. In order to overcome stability problems, several stabilizing techniques are introduced in the numerical procedure. Also, the stress component equations are solved using matrix block tridiagonal solution to enhance stability using non staggered grids.

To check the grid independence of the solution for Configuration 2, some crucial quantities for turbulent Taylor-Couette flows have been considered: the mean tangential velocity component at mid-plane for the mean field and the maximum of the turbulence kinetic energy in the whole cavity for the turbulent field. Table II shows that the $(N P R=180) \times(N P Z=400)$ mesh in the $(r, z)$ frame provides the best arrangement between accuracy and CPU time compared to the other meshes considered. For this grid, the size of the thinner mesh is $\Delta_{1} r=9 \times 10^{-8} h$ and $\Delta_{1} z=3.89 \times 10^{-6} h$ in the radial and axial directions respectively.

It is also verified that the grid is sufficiently refined close the cylinders to describe accurately the viscous sublayers. For example, the wall coordinate $r^{+}=\Delta_{1} r u^{*} / \nu\left(u^{*}\right.$ the friction velocity at the wall and $\Delta_{1} r$ the size of the first mesh in the radial direction) remains below 0.05 along both cylinders for configuration 2 , which is quite below the classical value $r^{+}=1$, for which the viscous sublayer is described by at least 5 


\begin{tabular}{c|c|c|c} 
difference with the $180 \times 400$ mesh & $180 \times 500$ & $180 \times 600$ & $200 \times 600$ \\
\hline \hline mean field & $1.8 \%$ & $2.2 \%$ & $1.4 \%$ \\
\hline turbulent field & $0.2 \%$ & $0.2 \%$ & $0.3 \%$
\end{tabular}

Table II: Influence of the mesh grid on the mean and turbulent fields for $L=0.013, R_{i}=10216$ and $C_{w}=14858$ (Configuration 2). Comparisons with the $180 \times 400$ mesh, which is chosen as the reference mesh grid.

mesh points.

\begin{tabular}{c|c|c|c|c} 
configuration & $N P R \times N P Z$ & $\Delta_{1} r / h$ & $\Delta_{1} z / h$ & $\max \left(r^{+}\right)$ \\
\hline \hline 1 & $180 \times 400$ & $5.65 \times 10^{-5}$ & $6.38 \times 10^{-3}$ & 0.21 \\
\hline 2 & $180 \times 400$ & $9 \times 10^{-8}$ & $3.89 \times 10^{-6}$ & 0.05
\end{tabular}

Table III: Computational details for both configurations. $\Delta_{1} r$ and $\Delta_{1} z$ are the size of the first cell in the radial and axial directions and $\max \left(r^{+}\right)$is the maximum value of the wall coordinate along both cylinders.

It has also been checked that the same grid arrangement provides a grid independent solution for the Configuration 1 corresponding to the experiments of Escudier and Gouldson [7]. Table III summarizes the computational details used in the present work. The $180 \times 400$ mesh in the $(r, z)$ frame has then proved to be sufficient to get grid-independent solutions for both configurations. It will be used for all cases considered in the following. About 30000 iterations (20 hours) on the M2P2 cluster composed of 2 xeon quadcore 3 $\mathrm{GHz}$ are necessary to obtain the numerical convergence of the calculations.

\section{Boundary conditions}

For both configurations, all the variables are set to zero at the walls except for the tangential velocity $V_{\theta}$, which is set to $\Omega R_{i}$ on the inner rotating cylinder and zero on the outer stationary cylinder. A linear profile for the mean tangential velocity component is imposed at the inlet as we the aspect ratio of the cavity is quite weak. Thus, $V_{\theta}$ varies linearly from zero on the outer wall up to $\Omega R_{i}$ on the inner wall. When a throughflow is enforced, a parabolic profile is then imposed for the axial velocity $V_{z}$ at the cavity inlet, with a given low level of turbulence intensity (1\%). In the outflow section, the pressure is permanently fixed, whereas the derivatives for all the other independent quantities are set to zero if the fluid leaves the cavity, and fixed 
external values are imposed if reversed flow occurs. A special treatment for this type of mixed boundary conditions has been developed. During the calculation, if reversed flow occurs, an advection equation for all quantity is solved in a region located just outside the physical domain (see Poncet [3]). It enables to moderate the evolution of these quantities and so to stabilize the calculations. Nevertheless, it is noteworthy that reversed flows have never been observed in the present work, whatever the values of the flow control parameters.

For Configuration 1, isothermal conditions are assumed. In Configuration 2, the temperature is fixed at the walls: $T=T_{i}$ at $r=R_{i}$ and $T=T_{o}$ at $r=R_{o}$. The temperature of the incoming fluid is fixed to $T_{a}$.

The velocity and thermal fields are initialized as follows: the fluid is at rest with a temperature of $T_{a}$.

\section{VALIDATION OF THE RSM MODEL IN THE CONFIGURATION 1 OF ESCUDIER AND GOULDSON [7]}

The predictions of the RSM model have already been widely validated in various rotating flow arrangements. Among others, we can cite the works on turbulent flows in a rotor-stator cavity with or without an imposed throughflow [1-5, 21], with heat transfer effects [6], and the work on the turbulent Von Kármán flow between counter-rotating disks equipped or not with straight blades [22]. Nevertheless, in order to show the detailed performances of the present model, our predictions are compared to the LDA measurements of Escudier and Gouldson [7] and to the $k-\varepsilon$ model of Naser [8] in a very elongated Taylor-Couette system defined by $L=0.0041$ and $s=0.506$. In this section, the rotational Reynolds number is fixed to $R e_{i}=961.07$ and the comparisons are performed at a given axial position $z^{*}=z / h=0.1$ for three values of the flow rate coefficient: $C_{w}=2839,5914,17742$. Thus, three values of the rotation parameter are considered: $N=0.968,0.465,0.148$.

As a preliminary, we define the following dimensionless quantities: the dimensionless radial $r^{*}=$ $\left(r-R_{i}\right) /\left(R_{o}-R_{i}\right)$ and axial $z^{*}=z / h$ positions. Thus, $r^{*}=0$ on the inner cylinder and $r^{*}=1$ on the outer cylinder. In the same way, $z^{*}=0$ corresponds to the cavity inlet and $z^{*}=1$ to the outlet. The mean tangential velocity component is normalized using the rotational speed of the inner cylinder $\Omega R_{i}$, whereas the mean axial velocity component is normalized using the mean axial velocity $\overline{V_{z}}$ imposed at the inlet, defined by $\overline{V_{z}}=Q /\left(\pi\left(R_{o}^{2}-R_{i}^{2}\right)\right): V_{\theta}^{*}=V_{\theta} /\left(\Omega R_{i}\right)$ and $V_{z}^{*}=V_{z} / \overline{V_{z}}$. To enable direct comparisons 
with the measurements of Escudier and Gouldson [7], the tangential $v_{\theta}^{\prime}$ and axial $v_{z}^{\prime}$ normal stresses are normalized by $\overline{V_{z}}: v_{\theta}^{\prime}=\sqrt{\overline{v_{\theta}^{\prime 2}}} / \overline{V_{z}}$ and $v_{z}^{*}=\sqrt{\overline{v_{z}^{\prime 2}}} / \overline{V_{z}}$.

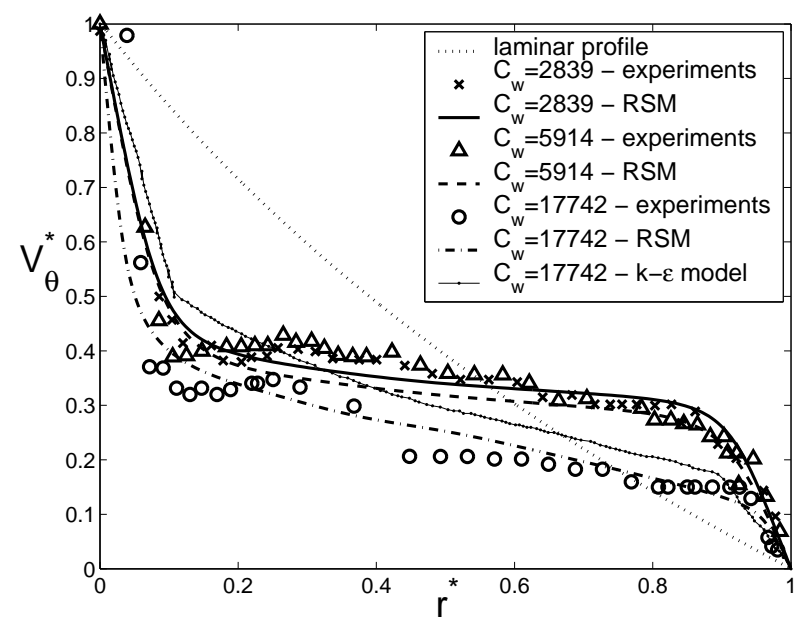

(a)

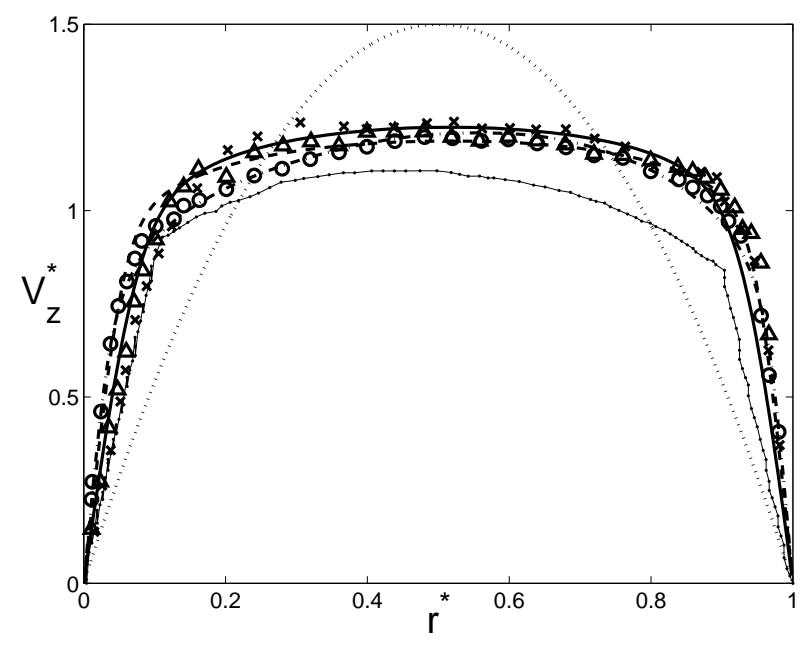

(b)

Figure 2: Radial distributions of the mean (a) tangential and (b) axial velocity components for $L=0.0041, s=0.506$ and $R e_{i}=961.1$ at $z^{*}=0.1$; Comparisons between the present RSM (lines) and the LDA measurements of Escudier and Gouldson [7] (symbols) for three values of $C_{w}: C_{w}=2839(\times,-), C_{w}=5914(\triangle,--)$ and $C_{w}=17742(\circ, .-)$. The laminar profiles (dotted lines) and the predictions of the $k-\varepsilon$ model of Naser [8] (dash-dotted) for $C_{w}=17742$ are also shown.

Figure 2 shows the distributions of the dimensionless mean tangential and axial velocity components at $z^{*}=0.1$. For the two lowest values of $C_{w}$, the tangential velocity profiles (Fig.2a) exhibit a structure divided into three regions: two thin boundary layers developed on each cylinder separated by a core rotating at a constant velocity. The central region rotates at $32 \%$ (resp. $34 \%$ ) of the cylinder speed for $\left(C_{w}=2839 ; N=\right.$ 0.968 ) (resp. $\left.\left(C_{w}=5914 ; N=0.465\right)\right)$ well below the value 0.53 given by Taylor [32] in the absence of throughflow. The flow is here mainly governed by the rotation for these values of $N$. A decrease of the rotation parameter to $N=0.148$ (corresponding to an increase of the flow rate coefficient to $C_{w}=17742$ ) implies a decrease of the rotating speed of the core region. Moreover, the tangential velocity is no more constant in the gap but is inversely proportional to the radius. Thus, the mean angular momentum is almost constant in that region.

There is only a weak effect of the flow rate coefficient on the radial distributions of the axial velocity (Fig.2b). The profiles are close to the turbulent Poiseuille flow profiles in pipes with a nearly constant axial 
velocity in the gap and thin boundary layers on the cylinders. For this value of radius ratio $s=0.506$, the profiles are almost symmetric. The profiles become flatter with increasing $N$ as already noted by Nouri and Whitelaw [15]. It is noteworthy that, whatever the value of $C_{w}$, the mean velocity profiles are far from the laminar profiles highlighting the turbulent nature of the flow.

For the mean field, the predictions of the RSM model are in very good agreement with the experimental data. The RSM predicts quite well the mean tangential velocity in the core of the flow. Moreover, it offers a good description of the boundary layer thicknesses along the cylinders as it can be seen from the mean axial velocity profiles. For $C_{w}=17742$, the RSM improves significantly the results of the $k-\varepsilon$ of Naser [8], which fails to predict the right profiles with large discrepancies for both the axial and tangential velocity components. The axial velocity is largely underestimated in the core and the tangential velocity is slightly overestimated. Fully developed conditions are reached at $z^{*}=0.1$ using the RSM in agreement with the observations of Escudier and Gouldson [7], whereas the predictions of the $k-\varepsilon$ model of Naser [8] showed a large dependence of the tangential velocity profiles on the axial position. The main reasons for these discrepancies are that the $k-\varepsilon$ model is blind to any rotation effects, and that the eddy viscosity concept, on which this model is based, is unsuitable with the present flow situation. On the contrary, the present RSM model is both sensitized to rotation effects $[1,21]$ and free from any eddy viscosity hypothesis.

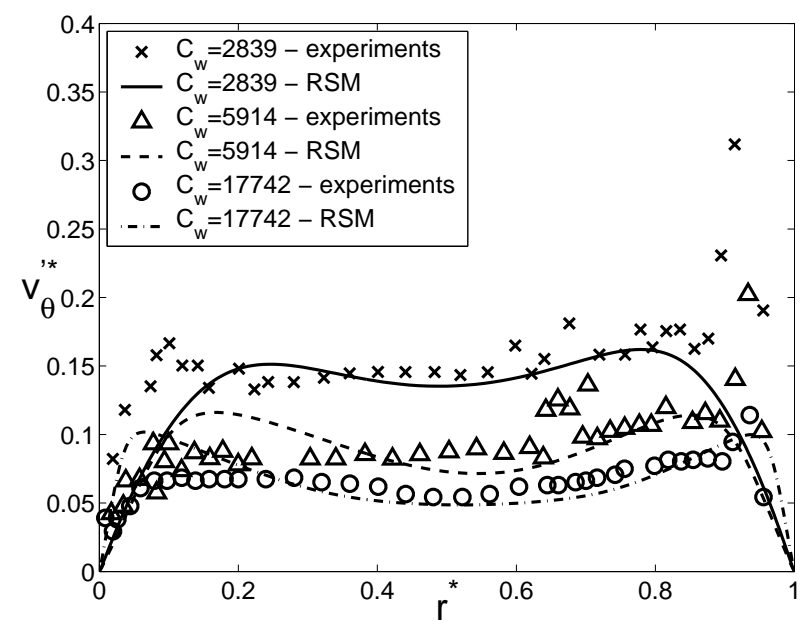

(a)

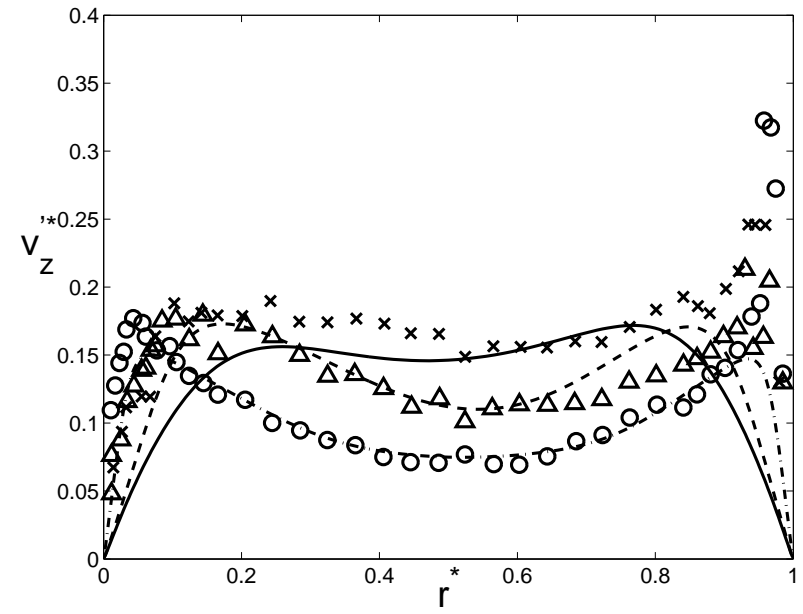

(b)

Figure 3: Radial distributions of the (a) tangential $v_{\theta}^{\prime *}$ and (b) axial $v_{z}^{\prime *}$ normal Reynolds stress tensor components. See legend of Figure 2. 
Figure 3 presents the radial distributions of the tangential and axial normal Reynolds stress tensor components for the same sets of parameters. Turbulence is mainly concentrated in the core region and vanishes towards the walls. The tangential and axial velocity fluctuations show a progressive decrease with increasing flow rate coefficient (or decreasing rotation parameter) in agreement with the experimental data of Escudier and Gouldson [7] and the LES results of Chung and Sung [20]. It is attributed by Escudier and Gouldson [7] to the vortical structures observed for low $C_{w}$ values induced by the centerbody rotation. For high values of $C_{w}$, the radial penetration of the rotational influence is reduced and turbulent fluctuations are suppressed as if there were no solid body rotation. The profiles of $v_{\theta}^{\prime *}$ and $v_{z}^{\prime *}$ are asymmetric for the highest value of the rotation parameter $N=0.968\left(C_{w}=2839\right)$ in agreement with [20], which can be attributed to the destabilizing effect of the centrifugal forces. All these phenomenons are well reproduced by the RSM, which predicts also quite good the turbulent intensities in the core of the flow. Some discrepancies are obtained in the boundary layers, especially for the peak values very close to the walls. The variations in the radial direction of the turbulent levels along each cylinder are also smoother than the experimental ones, which was also the case for the LES results of Chung and Sung [20] against the measurements of Nouri and Whitelaw [15].

Considering also the previous validations in various interdisk cavities [1, 3-6, 22], the second order model can now be used confidently to carry a parametric study of turbulent Taylor-Couette-Poiseuille flows with heat transfers in a narrow gap cavity characterized by a very small aspect ratio $L=0.013$ and a large radius ratio $s=0.961$ and for a wide range of the flow parameters (the Reynolds number $R e_{i}$, the flow rate coefficient $C_{w}$ and the Prandtl number $\left.P r\right)$.

\section{BASIC CASE IN CONFIGURATION 2}

Configuration 2 (see Table I) is now considered. Before performing a parametric study, a basic case $\left(R e_{i}=\right.$ 10216, $C_{w}=14858, N=4.24$ and $\left.\operatorname{Pr}=0.7\right)$ is investigated in details to fully depict the hydrodynamic and thermal fields. 


\section{A. Mean field}

Figure $4 \mathrm{a}$ and $4 \mathrm{~b}$ present the radial profiles of the mean tangential and axial velocity components respectively, at different axial positions $z^{*}$. The mean radial velocity component is not shown as it is quasi zero in the whole cavity. The mean flow is helical: the main flow is tangential due to the rotation of the inner cylinder (Taylor-Couette flow) and the secondary flow is essentially axial due to the superimposed throughflow (Poiseuille flow), which is confirmed by the streamline patterns shown in figure $6 \mathrm{a}$.

We recall that the boundary conditions imposed at $z^{*}=0$ correspond to the laminar regime with a linear Taylor-Couette profile for $V_{\theta}^{*}$ and a parabolic Poiseuille profile for $V_{z}^{*}$. From $z^{*} \simeq 0.2 \simeq 15.6 L$ to 0.8 , the mean flow is well established and the profiles do not depend anymore on the axial position $z^{*}$. The mean profiles of $V_{\theta}^{*}$ and $V_{z}^{*}$ are then characteristic of the turbulent regime with two very thin boundary layers developed on each cylinder. The central region between the two boundary layers is characterized by a quasi constant mean axial velocity equal to approximatively 1.07, close to a turbulent Poiseuille-like profile in pipes. The mean tangential velocity component increases linearly when moving from the outer to the inner cylinders in that region in agreement with the LES results of Chung and Sung [20]. This behavior is analogous to the turbulent torsional Couette flow found in very flat rotor-stator disk cavities [5]. $V_{\theta}^{*}$ is exactly equal to 0.5 at mid-radius, which is to be compared to the theoretical value 0.48 of Polkowski [33], whereas Kuosa et al. [19] obtained 0.083 with the $k-\omega$ SST model. Note that this swirl level is much higher than the measured or computed one obtained in the case of the Escudier and Gouldson's [7] experiment, which is a direct effect of both the narrow gap between the walls and to the high value of the rotation parameter $N=4.24$ considered here. From the radial profiles in figure 4 , we can deduce the thicknesses of the boundary layers $\delta_{V i}$ and $\delta_{V o}$ on the inner and outer cylinders respectively. $\delta_{V i}$ (resp. $\delta_{V o}$ ) is the height at which the mean tangential velocity component reaches $99 \%$ (resp. 1\%) of $\Omega R_{i}$. For $0.2 \leq z^{*} \leq 0.8$, these thicknesses are almost constant and equal to $\delta_{V i} /(\Delta R)=0.03$ and $\delta_{V o} /(\Delta R)=0.04$.

In the outlet section $\left(z^{*}=1\right)$ where a given pressure is imposed, absolutely no reversed flow has been observed in the present case. It can be seen also from the streamline patterns shown in Figure 6a. The small contraction of the streamlines at the outlet is only due to the apparent aspect ratio 0.345 of Figure $6 \mathrm{a}$, which does not respect the real aspect ratio of the cavity equal to 0.013 . At the outlet, $V_{\theta}^{*}$ varies non monotonously with $r^{*}$. Close to the inner rotating cylinder, it varies very quickly from 0.1 for $r^{*}=0.1$ to 1 
on the rotor. The axial velocity profile tends to the laminar profile at the outlet with a strong axial velocity in the center of the gap.

(a)

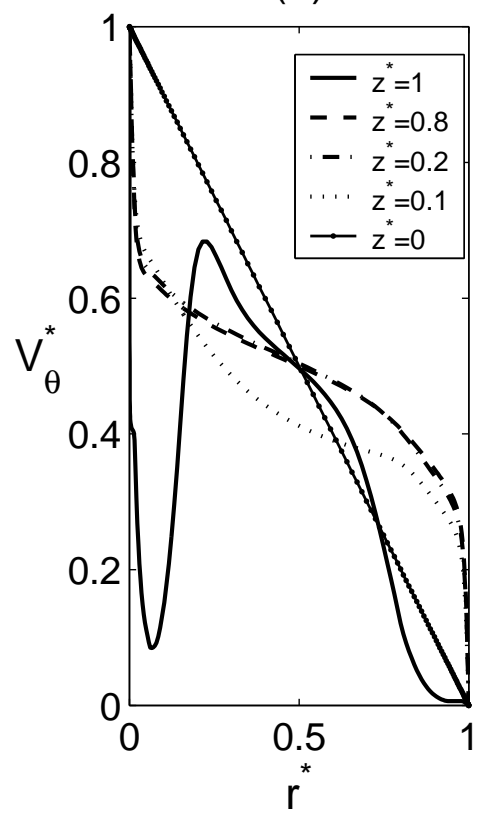

(b)

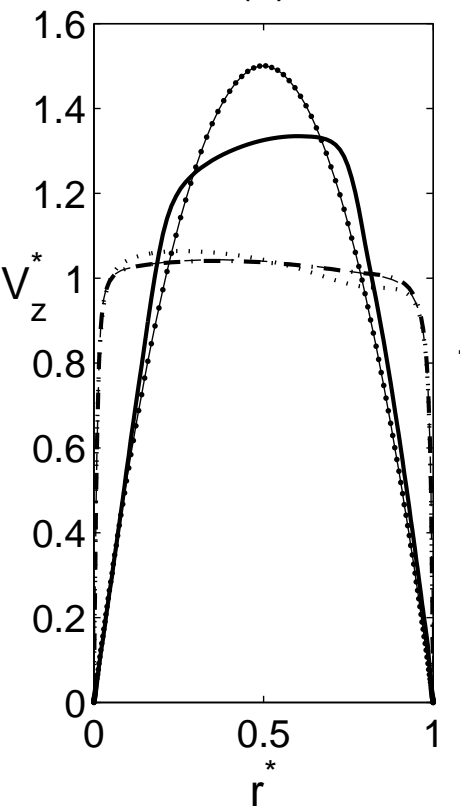

(c)

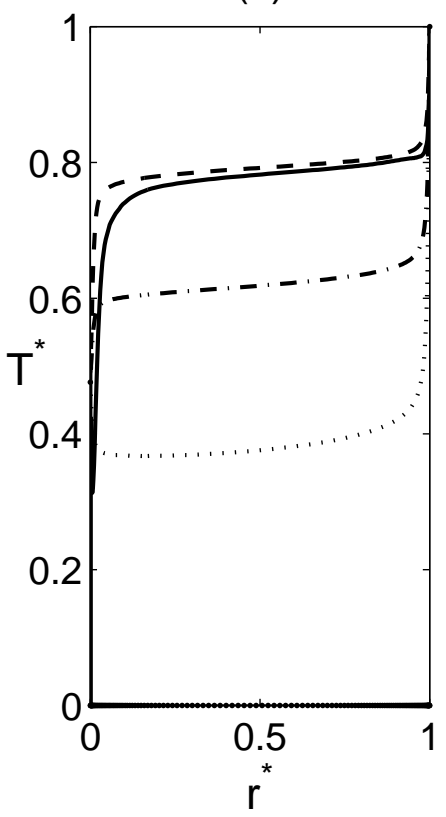

Figure 4: Radial profiles of the mean (a) tangential velocity component $V_{\theta}^{*}$, (b) axial velocity component $V_{z}^{*}$ and (c) dimensionless temperature $T^{*}$ for different axial positions $z^{*}$ and $R e_{i}=10216, C_{w}=14858$ and $P r=0.7$.

As for the hydrodynamic mean flow, the temperature field $T^{*}$ does not depend on the axial position for $z^{*} \geq 0.4$ (Fig.4c). Note that the temperature $T$ is normalized as follows: $T^{*}=\left(T-T_{a}\right) /\left(T_{o}-T_{a}\right)$. Thus, $T^{*}=0$ in the inlet section $\left(z^{*}=0\right)$ and $T^{*}=1$ along the outer cylinder $\left(r^{*}=1\right)$, where the highest temperature is reached (see also figure 6c). The mean temperature profiles can also be divided into three areas: two thermal boundary layers separated by a region, where the temperature is quasi constant. For $z^{*} \geq 0.4$, the temperature reached in the core region tends to $T^{*}=0.768$. It is slightly higher than the value $T^{*}=0.738$ corresponding to the average value between the cylinder temperatures $T_{i}$ and $T_{o}$. It shows in particular the dominating influence of the (warmest) outer cylinder. For this set of parameters, rotation effects on the mean flow are dominant compared to the superimposed throughflow as the value of the rotation parameter $N=4.24$ is high. Thus, the residence time of the incoming fluid (at $\left.T^{*}=0\right)$ inside the cavity is large enough for the walls to warm significantly the fresh incoming fluid. It explains why the mean temperature remains in the range $0.7 \leq T^{*} \leq 0.9$ (Figure $6 \mathrm{c}$ ). From the temperature profiles 
in figure $4 \mathrm{c}$, we can evaluate the thicknesses of the thermal boundary layers denoted $\delta_{T i}$ and $\delta_{T o}$ on the inner and outer cylinders respectively. $\delta_{T i}\left(\operatorname{resp} . \delta_{T o}\right)$ is the height at which the mean temperature reaches $99 \%$ (resp. $1 \%$ ) of the averaged temperature in the core. Thus, $\delta_{T i} / \Delta R=0.023$ and $\delta_{T o} / \Delta R=0.06$ for $0.4 \leq z^{*} \leq 0.8$. As expected, the thermal boundary layer is thicker than the hydrodynamic one along the stator. The Prandtl number, which compares the hydrodynamic and thermal boundary layer thicknesses, is indeed equal to $\operatorname{Pr}=0.7 \leq 1$ in the present case.

\section{B. Turbulent field}
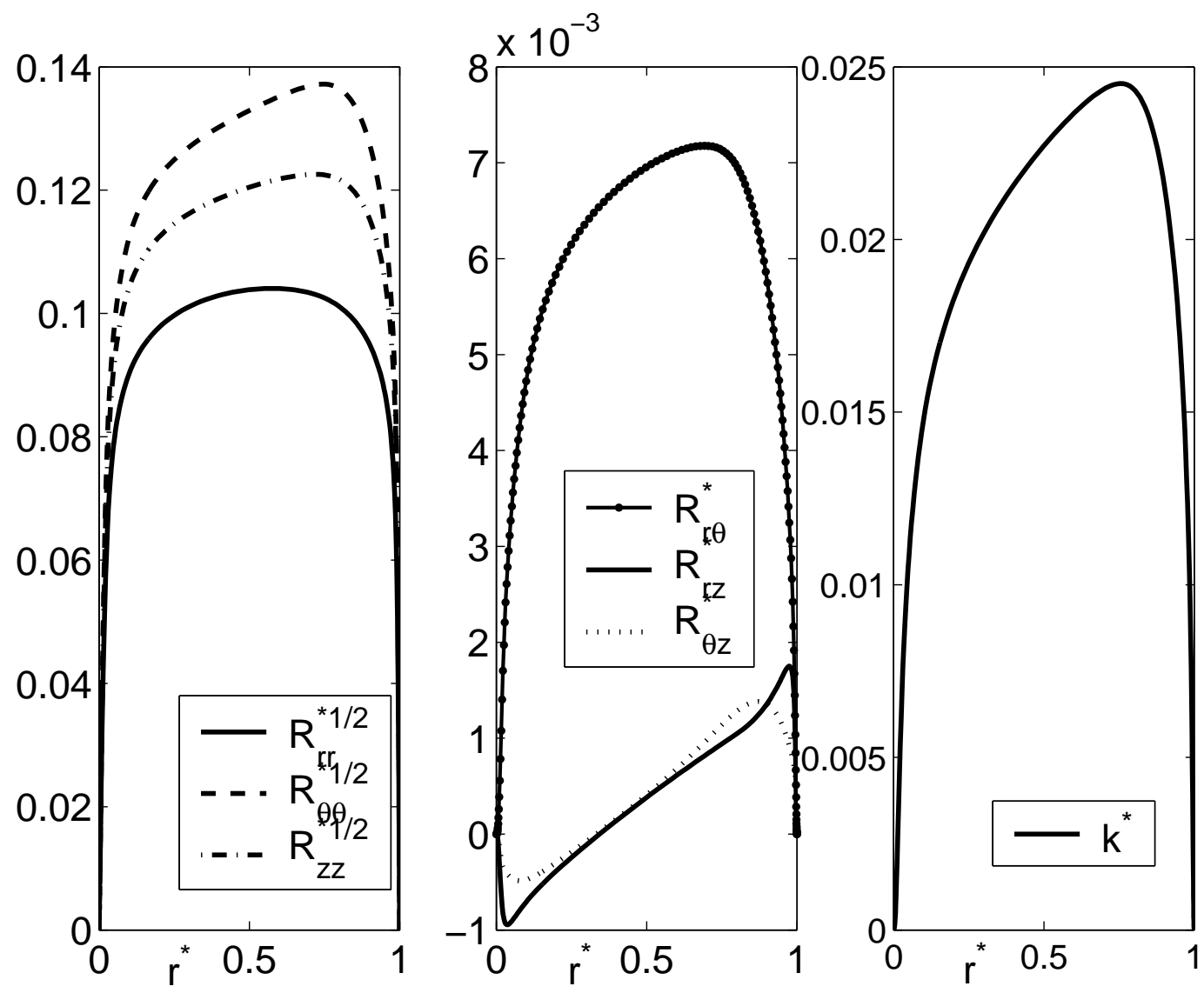

Figure 5: Radial profiles of the six Reynolds stress tensor components and of the turbulence kinetic energy for $R e_{i}=10216, C_{w}=14858$ and $\operatorname{Pr}=0.7$ at $z^{*}=0.5$.

Figure 5 shows the radial profiles of the six Reynolds stress tensor components $R_{i j}^{*}$ and of the turbulence kinetic energy $k^{*}$ at mid-height $z^{*}=0.5$ for the same set of parameters. Note that, in the following, the 
Reynolds stress tensor components as well as $k^{*}$ are normalized by $\left(\Omega R_{i}\right)^{2}$. It is not shown here but as for the mean field, there is no axial dependence of these turbulent quantities for $0.2 \leq z^{*} \leq 0.8$. Turbulence is then fully developed in that region and not influenced by the inlet and outlet areas. The highest levels of the normal Reynolds stress tensor components are reached in the core of the flow with maxima closest to the outer cylinder. As for the highest value of the rotation parameter $N=0.968$ considered in Configuration 1, it can be attributed to the destabilizing effect of the centrifugal forces. Turbulence intensities vanish at the walls. The magnitudes of the three normal Reynolds stress tensor components are quite comparable indicating that the turbulence is only weakly anisotropic. The $R_{r \theta}^{*}$ behaves like the normal components, with a maximum along the external cylinder. The two other cross components, which are one order of magnitude below, indicate a strong shear along the cylinders in agreement with the LES results of Chung and Sung $[20]$.

(a)

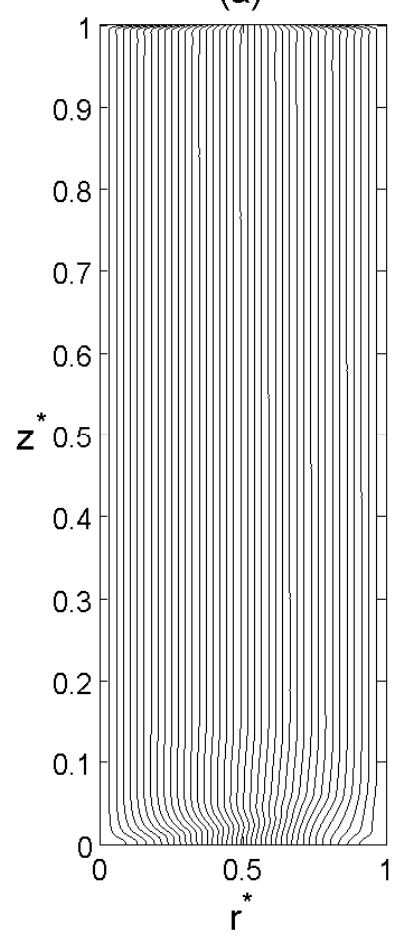

(b)

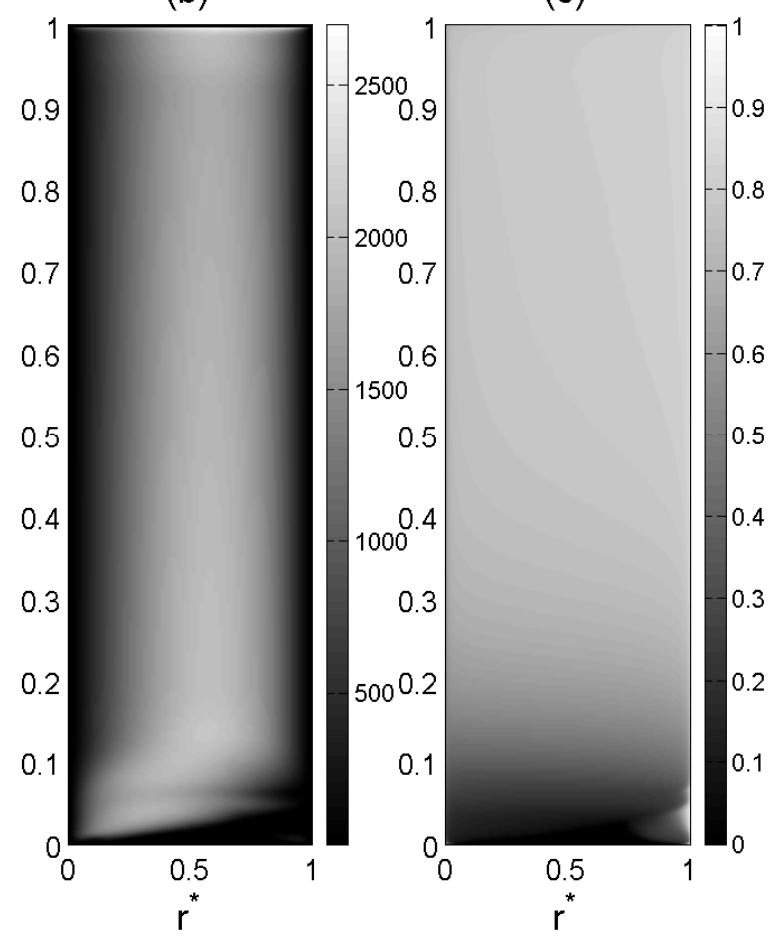

Figure 6: $R e_{i}=10216, C_{w}=14858$ and $\operatorname{Pr}=0.7$. Isovalues of: (a) the stream function $\Psi^{*}=\Psi /\left(\Omega R_{0}^{2}\right)$, (b) the turbulence Reynolds number $R e_{t}=k^{2} /(\nu \varepsilon)$, (c) the temperature $T^{*}$. Apparent aspect ratio equal to 0.345.

These last results on the turbulent field are confirmed by figures $6 c$ and 7 . The isovalues of the turbulence 
Reynolds number $R e_{t}=k^{2} /(\nu \varepsilon)$ (Fig.6c) enable to visualize the turbulent regions in the cavity. The flow is considered as being laminar for $R e_{t}=1 / c_{\mu} \leq 11\left(c_{\mu}=\nu_{T} \varepsilon / k^{2}=0.09\right.$, where $\nu_{T}$ is the turbulence viscosity). The flow in that case is turbulent everywhere apart from a very tiny region delimited by the equation $z^{*} \simeq 0.005+0.045 r^{*}\left(0 \leq r^{*} \leq 1\right)$ where $R e_{t} \leq 11$. In the core, the flow is highly turbulent as $R e_{t}$ can reach the maximum value of 2696 .

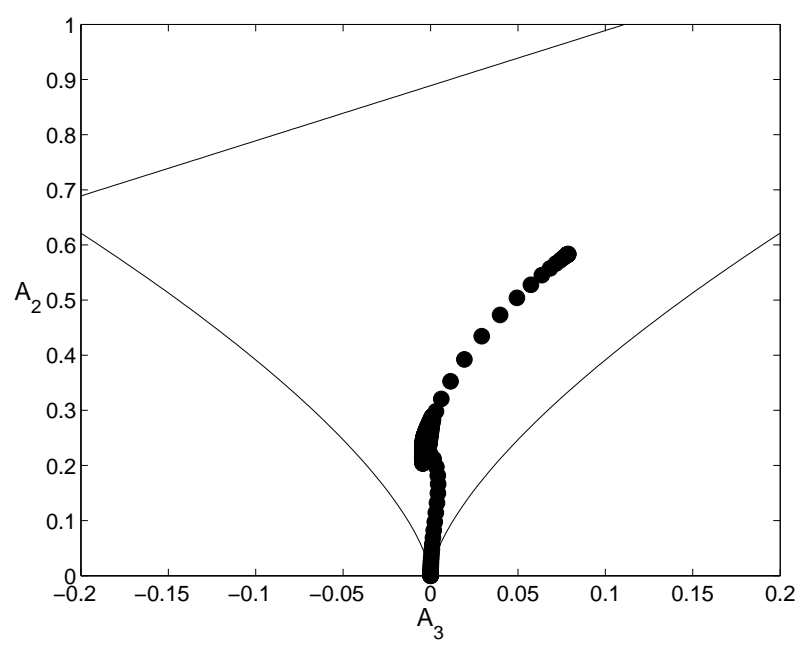

Figure 7: Anisotropy invariant map at $z^{*}=0.5$ for $R e_{i}=10216, C_{w}=14858$ and $\operatorname{Pr}=0.7$.

Figure 7 shows the anisotropy invariant map for the Reynolds stress tensor at $z^{*}=0.5$. The second $A_{2}$ and third $A_{3}$ invariants of the anisotropy tensor $a_{i j}$ of the second moments of the fluctuations are defined as $A_{2}=a_{i j} a_{j i}$ and $A_{3}=a_{i j} a_{j k} a_{k i}$ [34]. The results of the RSM model respect the realizability diagram of Lumley [34] as they remain within the region delimited by the two lines corresponding to the axisymmetric flow $A_{3}= \pm A_{2}^{3 / 2} / \sqrt{6}$ and the straight upper one corresponding to the two-component limit $A_{3}=A_{2}-8 / 9$. It confirms that the turbulence is almost isotropic in the core region as the curve tends to the origin point $\left(A_{2}=0, A_{3}=0\right)$. On the other way, the curve slightly tends to the one component limit (upper right corner in Figure 7) close to the walls.

\section{Turbulence kinetic energy budgets}

To highlight the influence of the additionnal terms taking into account the implicit effects of rotation on turbulence in the present RSM, budgets for the turbulence kinetic energy transport equation are here 
performed. The equation for the turbulence kinetic energy $k$ is given in [1] as:

$$
\frac{d k}{d t}=P+D^{T}+D^{\nu}-\epsilon+D^{R}+J
$$

The terms $D^{R}$ and $J$ model two implicit effects of rotation on turbulence. $D^{R}$ is an inhomogeneous diffusion term, which slows down the tendancy of bidimensionalization close to the walls. Its empirical term takes also into account the significant increase of the turbulent diffusion due to the triple fluctuating velocity correlation and to the fluctuating pressure in the case of strong rotation. Another characteristic phenomenon due to rotation is a reduction of the energy transfer from large to small turbulent scales. This last phenomenon is modeled here through an inverse flux $J$. All details about these terms can be found in [1].
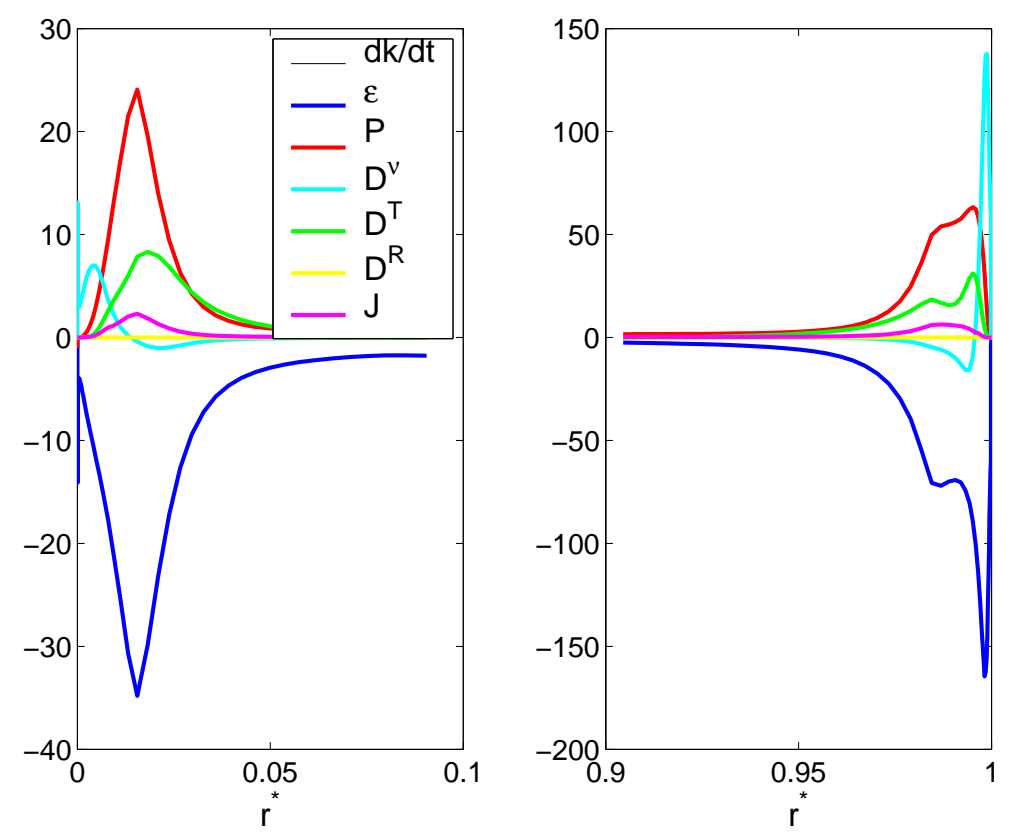

Figure 8: Turbulence kinetic energy budgets at $z^{*}=0.5$ along both cylinders for $R e_{i}=10216, C_{w}=14858$ and $\operatorname{Pr}=0.7$.

Budgets for the turbulence kinetic energy $k$ (normalized here by $(\Omega h)^{2}$ ) are presented in Figure 8 at midheight of the cavity in both boundary layers. The transport of $k$ is mainly governed by the production $P$ and the dissipation $\epsilon$ terms, which compensate almost each other. The molecular $D^{\nu}$ and turbulent $D^{T}$ diffusion terms can not be neglected close to the walls. In the viscous sublayers, where low Reynolds numbers are reached, $D^{\nu}$ compensates exactly the dissipation $\epsilon$, whereas the term $D^{T}$ is almost zero. The inhomogeneous 
diffusion term $D^{R}$, which usually flattens the turbulence kinetic energy by diffusion along the rotation axis [3], does not contribute here to the $k$ budgets. The inverse flux $J$, which increases the turbulence level in the core of the flow, has only a weak contribution to the $k$ budgets in both boundary layers, which does not mean that it does not affect the mean and turbulent fields. It can be noticed that the values reached along the stationary outer cylinder are much higher (in absolute value) than those obtained along the inner rotating one. It confirms in particular the RSM predictions of Poncet [3] in the case of rotor-stator disk flows with throughflow.

The influence of the additionnal terms contained in $T$ has been already addressed by Elena and Schiestel [1] for rotor-stator disk flows. They compared the predictions of three low-Reynolds number RSM models from the basic model of Hanjalic and Launder [35] to the final version described here. The introduction of the new terms did not produce important changes. Nevertheless, the final model was found to improve the predictions of the former ones. The turbulence levels close to the rotor and the location of the relaminarized and turbulent regions were in particular better predicted.

\section{Distributions of the local Nusselt numbers}

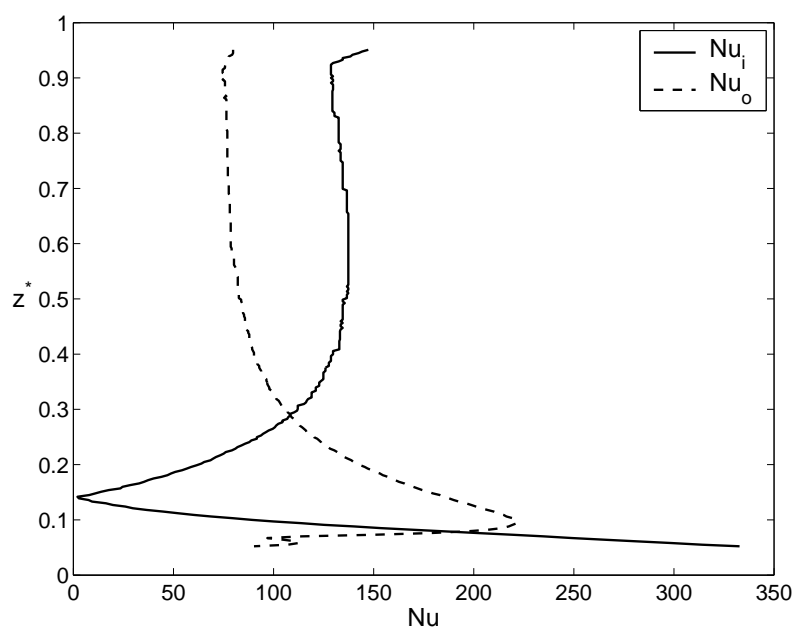

Figure 9: Axial distributions of the local Nusselt numbers along both disks for $R e_{i}=10216, C_{w}=14858$ and $\operatorname{Pr}=0.7$.

From the isotherm maps shown in figure 6c, we can deduce the axial distributions of the local Nusselt numbers $N u_{i}$ and $N u_{o}$ along the inner and outer cylinders respectively defined in equations (1) and (2). In 
figure 9 , we can notice strong variations of $N u_{i}$ and $N u_{o}$ for $z^{*} \leq 0.4$. For $z^{*} \geq 0.4$, as the mean temperature does not depend anymore on the axial location $z^{*}$, the local Nusselt numbers are nearly constant. $N u_{i}$ tends to 0 for $z^{*}=0.15$ as the incoming fluid warms and reaches the same temperature than the inner cylinder at this axial location. At $z^{*} \simeq 0.15, N u_{o}$ reaches a maximum, because the incoming cold fluid is ejected to the outer cylinder due to the centrifugal force and so keeps a relatively low temperature. Giret [9] did not measure the heat transfer coefficients close to the inlet where the strong variations are expected. Nevertheless, he observed also a strong decrease of $N u_{i}$ from $z^{*} \simeq 0.15$ to $z^{*} \simeq 0.8$.

\section{INFLUENCE OF THE FLOW PARAMETERS}

After having depicted the hydrodynamic and thermal fields for a basic case, 18 calculations have been performed to investigate the influence of the flow control parameters: the rotational Reynolds number $R e_{i}$, the flow rate coefficient $C_{w}$ and the Prandtl number $\operatorname{Pr}$.

\section{A. Hydrodynamic field}

The influences of the rotational Reynolds number $R e_{i}$ and the flow rate coefficient $C_{w}$ on the mean velocity profiles are shown in Figure 10 at mid-height for $3744 \leq R e_{i} \leq 37443$ and $0 \leq C_{w} \leq 30000$.

For a given flow rate $C_{w}=5000$, the tangential velocity is inversely proportional to the radius for the lowest value of $R e_{i}=3744$ corresponding to a rotation parameter $N$ equal to 4.61 (Fig.10a). It tends to the classical laminar profile in Taylor-Couette flow. For increasing values of $R e_{i}$ (or $N$ ), the tangential velocity at mid-radius decreases. A center body rotation progressively appears and is combined with a thinning of the boundary layers. The core region rotates at approximately $46 \%$ of the inner cylinder speed for $R e_{i}=37443$ $(N=46.14)$ and $V_{\theta}^{*}$ is constant over a large radial extension. In the same time, the radial distributions of the axial velocity (Fig.10b) flatten even the influence of the rotation is quite weak for this range of parameters.

The rotational Reynolds number is now fixed to $R e_{i}=10216$ and the flow rate coefficient varies between 0 ( $N$ tends to infinity) and $C_{w}=3 \times 10^{4}(N=2.1)$. The influence of $C_{w}$ on the mean velocity profiles remains weak for this set of parameters (Fig.10c,d). It shows in particular that the rotation parameter does not play any crucial role on the flow structure for the range of parameters considered here. 


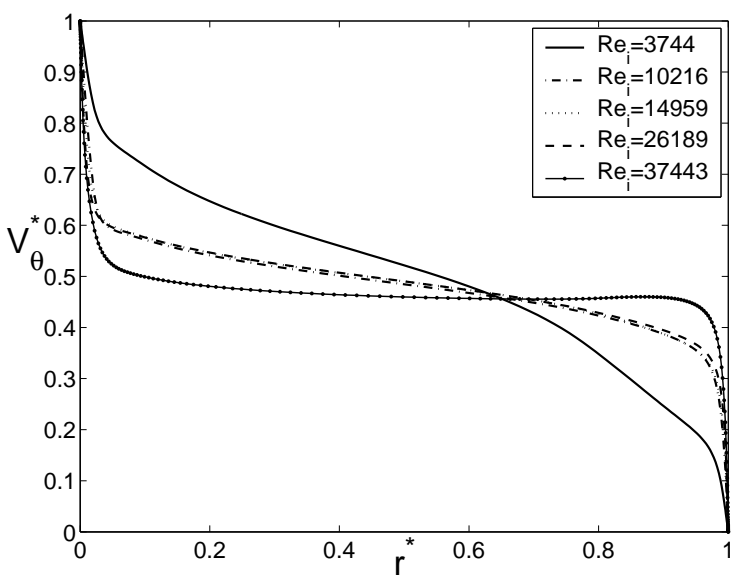

(a)

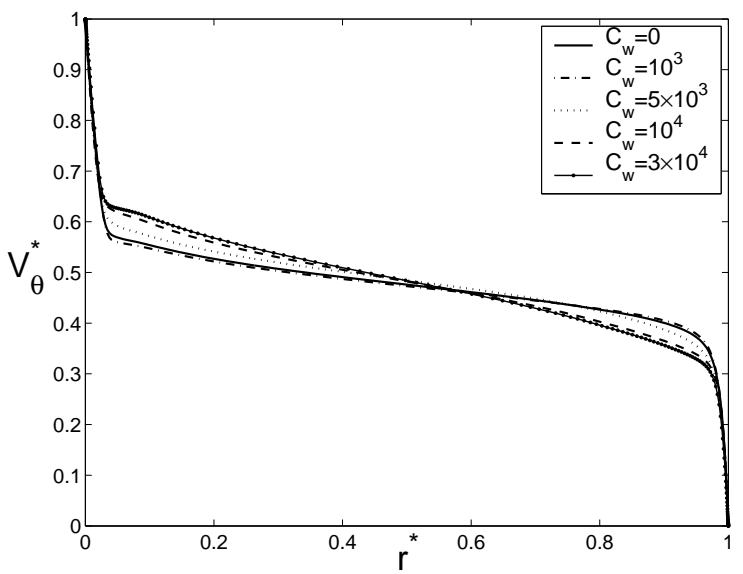

(c)

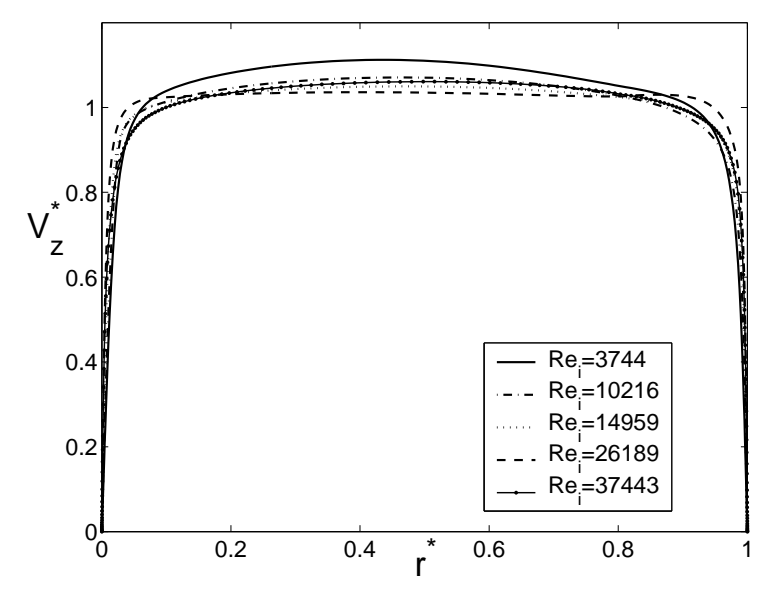

(b)

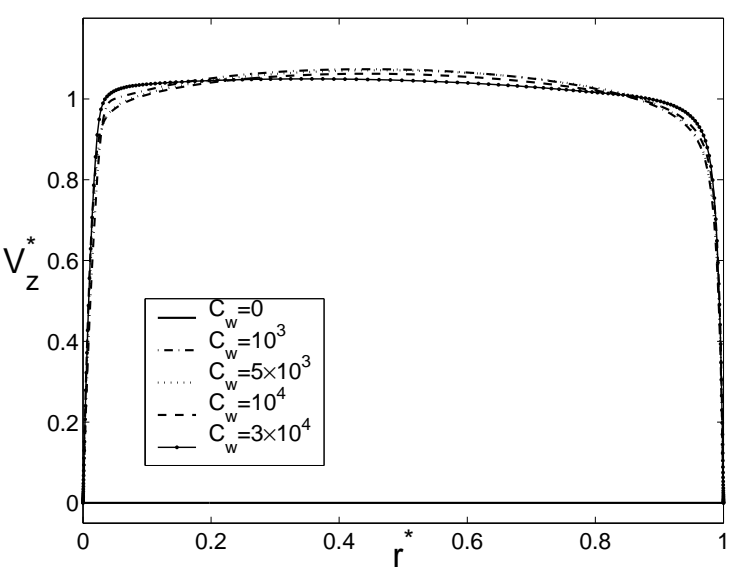

(d)

Figure 10: Influence of the Reynolds number $R e_{i}$ on the mean (a) tangential and (b) axial velocity components for $\operatorname{Pr}=0.7$ and $C_{w}=5000$ at $z^{*}=0.5 ;$ Influence of the flow rate coefficient $C_{w}$ on the mean (a) tangential and (b) axial velocity components for $\operatorname{Pr}=0.7$ and $R e_{i}=10216$ at $z^{*}=0.5$.

More interesting is the effect of the Reynolds number on the turbulent field illustrated by the radial distributions of the turbulence kinetic energy $k^{*}$ at mid-height $z^{*}=0.5$ (Fig.11a). Whatever the value of $R e_{i}$, the turbulence is concentrated in the core of the flow with slightly higher turbulence intensities on the outer cylinder side and vanishes towards the walls. The normalized turbulence kinetic energy $k^{*}=k /\left(\Omega R_{i}\right)^{2}$ decreases for increasing values of the Reynolds number, because of the normalization by $\left(\Omega R_{i}\right)^{2}$. As expected, $k$ is an increasing function of the rotation rate but it appears that, above a certain threshold, $R e_{i}$ has only a weak influence on the turbulence intensities, whereas $\left(\Omega R_{i}\right)^{2}$ still increases. 


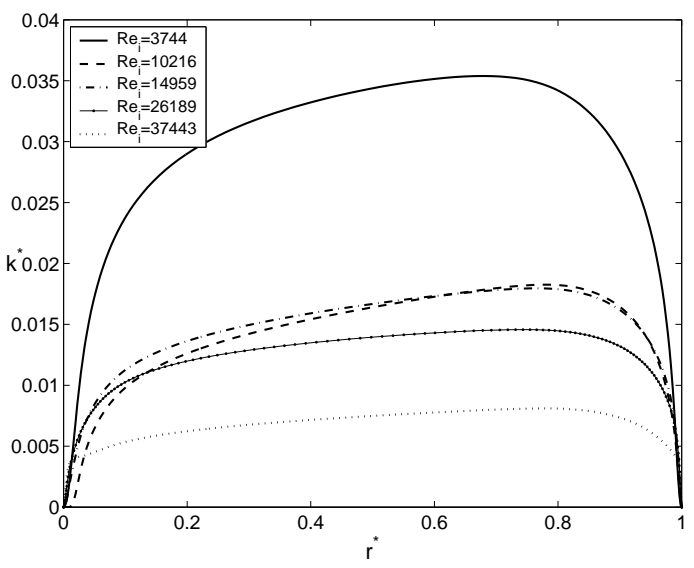

(a)

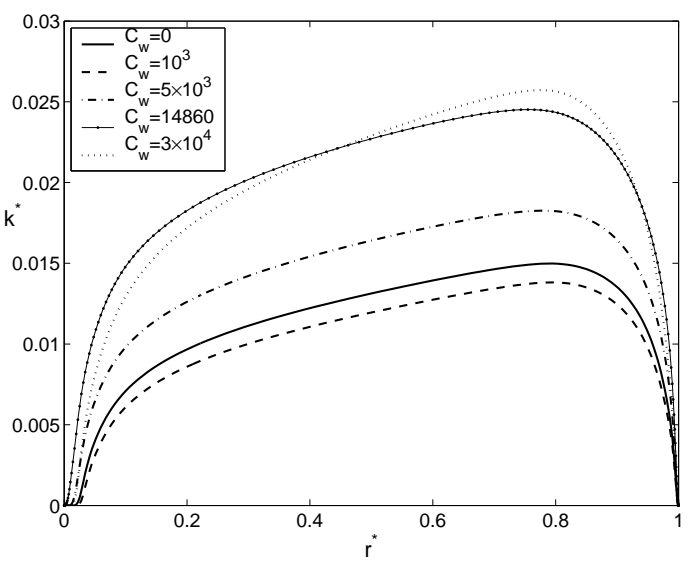

(b)

Figure 11: Radial distributions of the turbulence kinetic energy $k^{*}$ for $z^{*}=0.5$ and $\operatorname{Pr}=0.7$ : (a) Influence of the Reynolds number $\left(C_{w}=5000\right)$ and (b) influence of the flow rate coefficient $\left(R e_{i}=10216\right)$.

The effect of the superimposed throughflow on the turbulent field is presented in figure $11 \mathrm{~b}$ for $0 \leq C_{w} \leq$ 30000 also at mid-height $z^{*}=0.5$. The shape of the radial profiles remains the same. The turbulence kinetic energy $k^{*}$ increases with increasing values of $C_{w}$. The superimposed throughflow acts as the rotation rate on the turbulence. It can be explained by considering the effective Reynolds number $\operatorname{Re}_{e}=V_{e} \Delta R / \nu$, which is based on the efficient velocity $V_{e}=\sqrt{{\overline{V_{z}}}^{2}+\alpha\left(\Omega R_{i}\right)^{2}}$, where $\alpha$ is a weighting coefficient fixed in general to $0.25[14,36,37]$. Note that this value is obtained by supposing that the tangential velocity of the fluid in the gap is half the peripheral velocity of the inner cylinder for narrow gap cavity. This increase is less noticeable for low values of $C_{w}\left(0 \leq C_{w} \leq 2500\right)$ because, in this range, the mean and turbulent flow fields are mainly dominated by the rotation of the inner cylinder.

\section{B. Thermal field}

Figure 12 presents the isotherm maps in a $(r, z)$ plane for all the cases considered here highlighting the influence of the flow parameters on the thermal field. The effect of the Reynolds number is illustrated by comparing figures $12 \mathrm{a}$ to $12 \mathrm{e}$ for $C_{w}=5000$ and $\operatorname{Pr}=0.7$. For a given superimposed throughflow, when the rotation rate of the inner cylinder is increased, the centrifugal effect increases. The main flow is still helical but the thread of the screw gets even smaller. As a consequence, the time of residence increases with increasing 
values of $R e_{i}$, which implies a more efficient mixing and a better homogenization of the mean temperature in the whole cavity. So, the mean temperature in the center of the cavity $T_{\text {mid }}^{*}=T^{*}\left(r^{*}=0.5, z^{*}=0.5\right)$ is a decreasing function of $R e_{i}$ following the quadratic law: $T_{m i d}^{*}=7.5 \times 10^{-11} R e_{i}^{2}-6.4 \times 10^{-6} R e_{i}+0.88$. For very high Reynolds numbers, $T_{m i d}^{*}$ tends to the value 0.738 corresponding to the average between the wall temperatures. For all cases, the isotherms are parallel to the rotation axis. The size of cold fluid around the inlet increases with the rotation rate of the hub.

The effect of the flow rate coefficient on the temperature field (Fig.12f-12j) is more noticeable than the one of the Reynolds number. Without any superimposed throughflow (Fig.12f), the isotherms are parallel to the rotation axis and $T_{m i d}^{*}$ is equal to 0.841 , which is much warmer than the average temperature 0.738 between the two walls, showing the preponderant effect of the outer cylinder. When the flow rate coefficient increases, the isotherms get progressively inclined. For the highest value $C_{w}=30000$ (Fig.12j), the effect of $C_{w}$ is preponderant compared to the effect of the rotation rate $(N=2.1) . T_{m i d}^{*}$ appears to be a decreasing function of $C_{w}$ following the quadratic law: $T_{m i d}^{*}=-1.3 \times 10^{-10} C_{w}^{2}-2.6 \times 10^{-6} C_{w}+0.84$. It is simply due to the fact that the fresh incoming fluid spends a shorter time in the cavity when $C_{w}$ is high $(N$ is low).

We now investigate the influence of the Prandtl number $\operatorname{Pr}$ on the thermal field for $\operatorname{Re}_{i}=10216, C_{w}=$ 14858 and $0.01 \leq P r \leq 12 . \operatorname{Pr}=0.01$ is a typical value for liquid metals, $\operatorname{Pr}=0.7-1$ for gases $(\operatorname{Pr}=0.71$ for air at $20^{\circ} \mathrm{C}$ ) and $\operatorname{Pr}=2-12$ for water. For $\operatorname{Pr}=0.01$ (Fig.12k), the heat transfer process is dominated by conduction from the outer to the inner cylinder. For this Prandtl number, the fluid behaves thermally like a solid. The isotherms are then parallel to the rotation axis except very close to the inlet region. The mean temperature increases linearly from the hub to the shroud. When the Prandtl number $\operatorname{Pr}=\nu / \sigma$ increases (Fig.12l - 120), the thermal diffusivity of the fluid $\sigma$ decreases. Thus, the characteristic time for the heat transfer between the cylinders and the fluid increases. As the residence time of the fluid in the cavity remains the same $\left(R e_{i}\right.$ and $C_{w}$ being constant), the fluid requires more time to exchange heat with the walls. The thermal effects diminish then with increasing values of $\operatorname{Pr}$ and the flow behavior is essentially hydrodynamic. That is the reason why the mean temperature in the center of the cavity $T_{m i d}^{*}$ decreases for increasing values of $\operatorname{Pr}$ following the quadratic law: $T_{m i d}^{*}=0.0014 \operatorname{Pr}^{2}-0.052 \operatorname{Pr}+0.79$. 
(a)

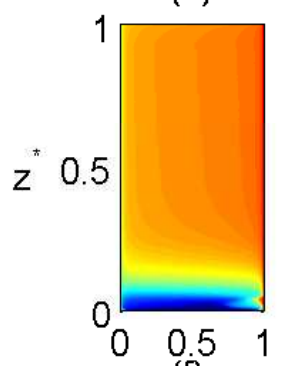

(f)

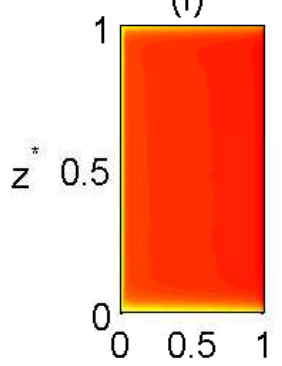

(k)

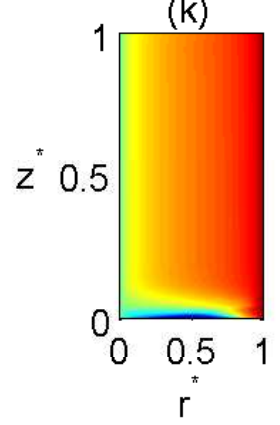

(b)

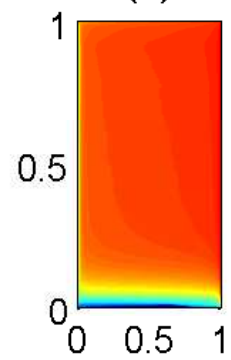

(g)

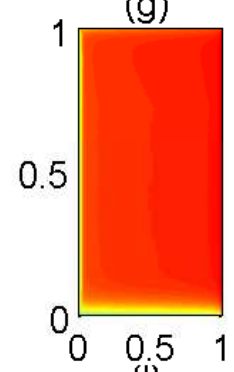

(i)

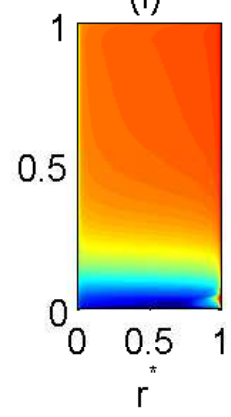

(c)

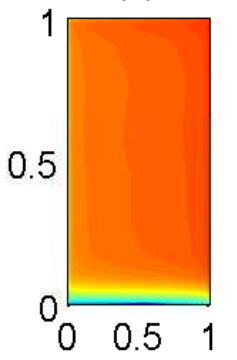

(h)

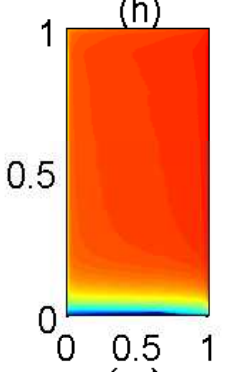

(m)

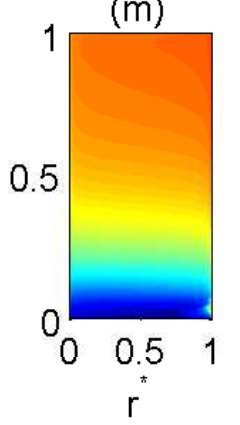

(d)

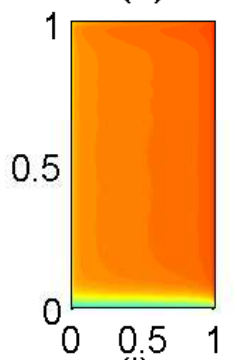

(i)
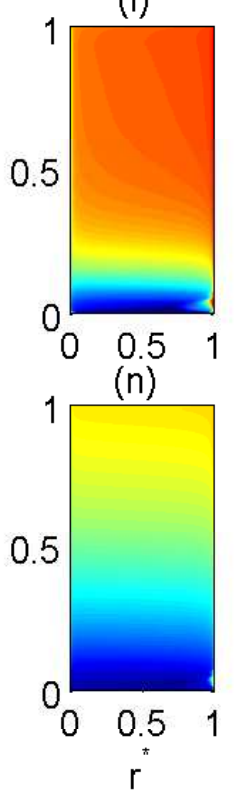

(e)
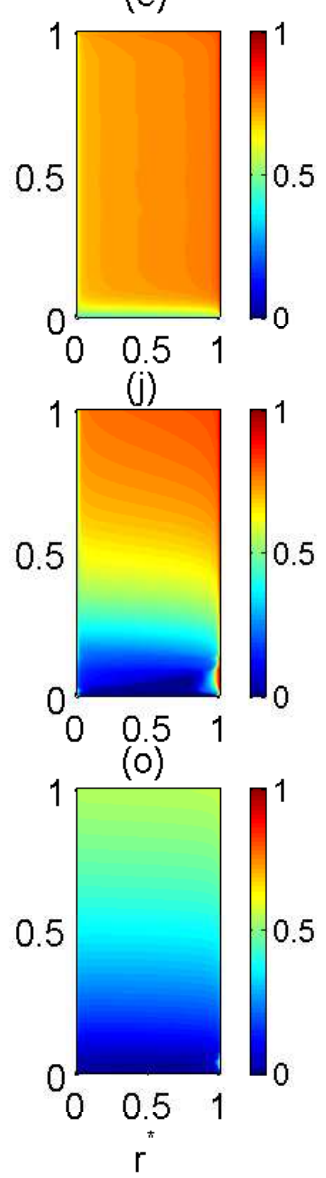

Figure 12: Isovalues of the mean temperature $T^{*}$.

Influence of the Reynolds number for $C_{w}=5000, \operatorname{Pr}=0.7$ : (a) $\operatorname{Re} e_{i}=3744$, (b) $R e_{i}=10216$, (c) $\operatorname{Re} e_{i}=14959$, (d) $R e_{i}=26189,(\mathrm{e}) R e_{i}=37443$.

Influence of the flow rate coefficient for $R e_{i}=10216, \operatorname{Pr}=0.7$ : (f) $C_{w}=0$, (g) $C_{w}=10^{3}$, (h) $C_{w}=5 \times 10^{3}$, (i) $C_{w}=14858,(\mathrm{j}) C_{w}=3 \times 10^{4}$.

Influence of the Prandtl number $\operatorname{Pr}$ for $R e_{i}=10216, C_{w}=14858:(\mathrm{k}) \operatorname{Pr}=0.01$, (l) $\operatorname{Pr}=0.7,(\mathrm{~m}) \operatorname{Pr}=2,(\mathrm{n})$ $\operatorname{Pr}=7,($ o) $\operatorname{Pr}=12$.

\section{Correlations}

These 18 sets of parameters cover a wide range of the rotation parameter $N$ : from 2.1 up to infinity, when no throughflow is superimposed. Thus, for all cases, the parameter $N$ is higher than 0.8 , which ensures that the Nusselt numbers depend on both the rotation $\Omega$ and the axial throughflow $Q$ (see in [13]). 


\section{Local Nusselt numbers}

The axial distributions of the local Nusselt numbers along the inner cylinder $N u_{i}$ and the outer one $N u_{o}$ are presented in figure $13 \mathrm{a}$ for $C_{w}=5000, \operatorname{Pr}=0.7$ and different Reynolds numbers. For this flow rate, $N u_{i}$ tends to zero for $z^{*} \simeq 0.1$ only for the smallest value of $R e_{i}=3744$. For the other Reynolds numbers, $N u_{i}$ increases with $z^{*}$ in the region close to the inlet and then, for $z^{*} \leq 0.2, N u_{i}$ remains almost constant. Along the outer cylinder, $N u_{o}$ decreases with $z^{*}$ at the cavity inlet and then remains also constant along the rest of the cylinder height. Except very close to the inlet, $N u_{i}$ and $N u_{o}$ increase for increasing values of the Reynolds number $R e_{i}$, which is expected in such rotating flows [38]. Figure $13 \mathrm{~b}$ sums up the results presented in figure 13a at a given axial position $z^{*}=0.5$, and confirms the dependence of $N u_{i}$ and $N u_{o}$ on the rotational Reynolds number $R e_{i}$.

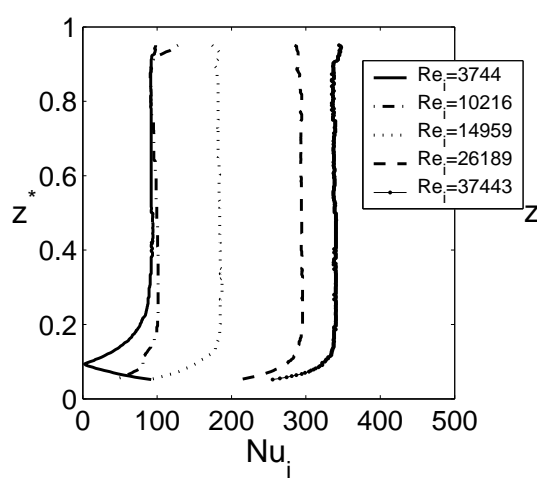

(a)

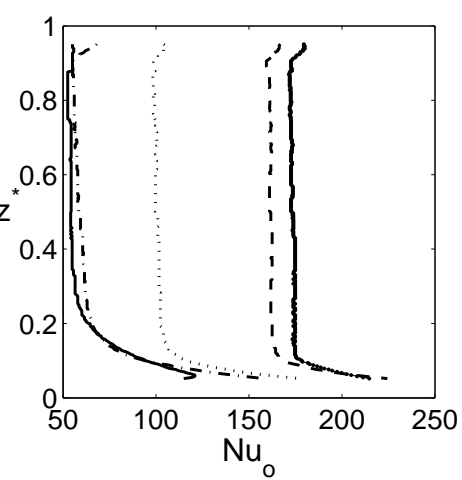

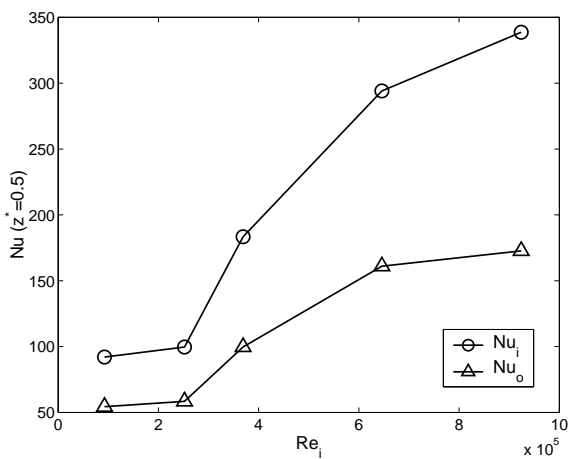

(b)

Figure 13: Local Nusselt numbers along both cylinders for $C_{w}=5000, \operatorname{Pr}=0.7$ and different Reynolds numbers $R e_{i}:$ (a) Axial profiles; (b) Variations against $R e_{i}$ at mid-height $z^{*}=0.5$.

The influence of the axial Poiseuille flow on the local Nusselt numbers is depicted in figure $14 \mathrm{a}$ for $R e_{i}=$ 10216, $\operatorname{Pr}=0.7$ and different values of the flow rate coefficient. Along the hub and for $C_{w} \leq 5000$, the local Nusselt number $N u_{i}$ does not depend on the axial location for $0.05 \leq z^{*} \leq 0.95$. $N u_{i}$ depends weakly on $C_{w}$ for $C_{w}<5000$ then increases strongly for higher flow rates. For $C_{w} \geq 10^{4}$, when $C_{w}$ increases, the axial location for which $N u_{i}$ tends to 0 moves towards the cavity outlet: $N u_{i} \simeq 0$ at $z^{*} \simeq 0.095$ for $C_{w}=10^{4}$, at $z^{*} \simeq 0.14$ for $C_{w}=14858$ and at $z^{*} \simeq 0.27$ for $C_{w}=30000$. In the same time, the region where $N u_{i}$ is quasi constant gets always smaller for increasing values of $C_{w}$. Along the stationary cylinder, the same behavior is observed. For $C_{w}<5000, N u_{o}$ depends very weakly on both $z^{*}$ and $C_{w}$. When increasing $C_{w}$ 
up to $30000, N u_{o}$ reaches a maximum close to the cavity inlet. This maximum is shifted to larger $z^{*}$ values, when $C_{w}$ increases. Thus, $N u_{o}$ is maximum at $z^{*} \simeq 0.06$ for $C_{w}=10^{4}$, at $z^{*} \simeq 0.1$ for $C_{w}=14858$ and at $z^{*} \simeq 0.17$ for $C_{w}=30000$. Note that the axial positions of the maxima of $N u_{o}$ do not coincide with the locations of the minima reached by $N u_{i}$ because of the helicity of the main flow. Figure $14 \mathrm{~b}$ confirms these previous results at a given location $z^{*}=0.5 . N u_{i}$ and $N u_{o}$ both increase for increasing values of $C_{w}$. The local decrease of $N u_{i}$ or $N u_{o}$ correspond to the fact that the fluid gets close the wall temperatures at this given location. Except for $C_{w}=30000$, the heat transfer coefficient along the rotating cylinder is higher than the one along the stationary cylinder at $z^{*}=0.5: N u_{i}>N u_{o}$.

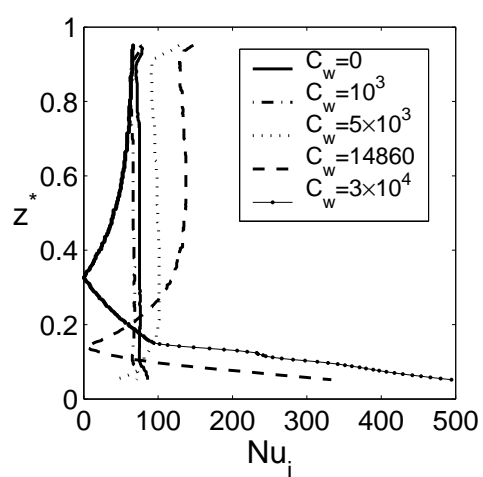

(a)

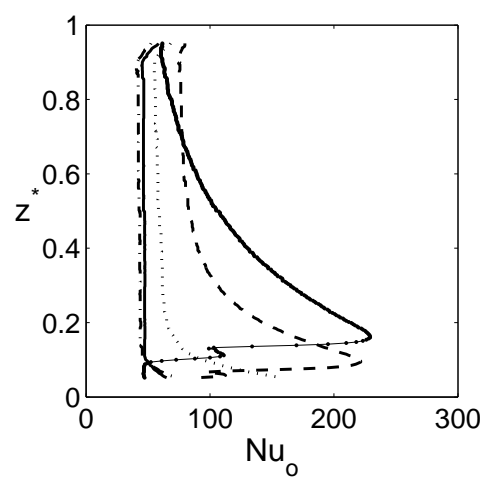

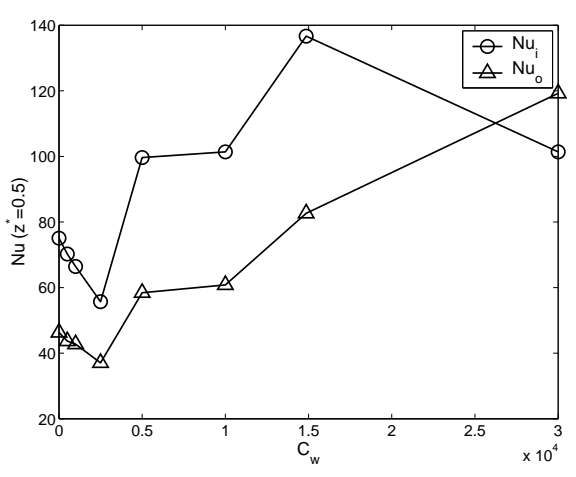

(b)

Figure 14: Local Nusselt numbers along both cylinders for $\operatorname{Re}_{i}=10216, \operatorname{Pr}=0.7$ and different values of the flow rate coefficient $C_{w}$ : (a) Axial profiles; (b) Variations at mid-height $z^{*}=0.5$ against $C_{w}$.

Figure 15a shows the axial distributions of the local Nusselt numbers for $R e_{i}=10216, C_{w}=14858$ and different Prandtl numbers. For liquid metals $(\operatorname{Pr}=0.01)$, both $N u_{i}$ and $N u_{o}$ are quite low and remain independent of $z^{*}$, which reflects the dominating influence of the molecular diffusivity on the heat transfer process. For higher Prandtl numbers $\operatorname{Pr} \geq 0.7, N u_{i}$ decreases for increasing values of $z^{*}$, reaches a minimum value at a given axial location and then increases weakly when moving towards the cavity outlet. The minimum value is obtained when the fluid is at approximately the same temperature than the wall. The axial location for which $N u_{i}$ reaches this minimum value is shifted towards the outlet for increasing Prandtl numbers. Along the outer cylinder, $N u_{o}$ increases for increasing $\operatorname{Pr}$ values. For gases $(\operatorname{Pr}=0.7)$ and liquids $(\operatorname{Pr}=[2,7,12]), N u_{o}$ reaches a maximum close to the cavity inlet and diminishes for larger axial locations. These results are confirmed by figure 15b, highlighting the influence of $\operatorname{Pr}$ on the local Nusselt number at 
mid-height. It clearly shows that $N u_{o}$ strongly increases for increasing $\operatorname{Pr}$ values and also that, for a given Prandtl number, $N u_{o}$ is larger than $N u_{i}$. Nu $u_{i}$ does not vary monotonously with $P r$, because at mid-height and for $\operatorname{Pr}=7$, the fluid reaches the wall temperature and so $N u_{i}$ tends to zero. Apart from $\operatorname{Pr}=7, N u_{i}$ slightly increases with $\operatorname{Pr}$.

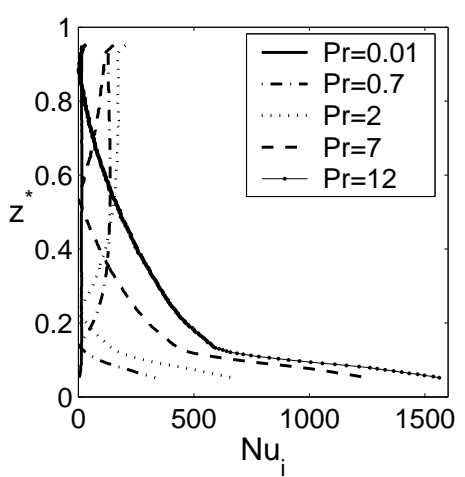

(a)

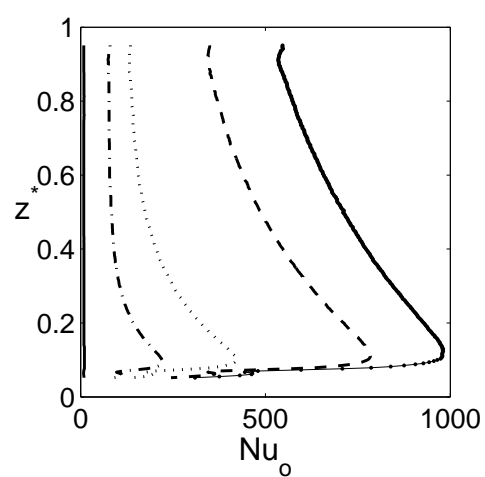

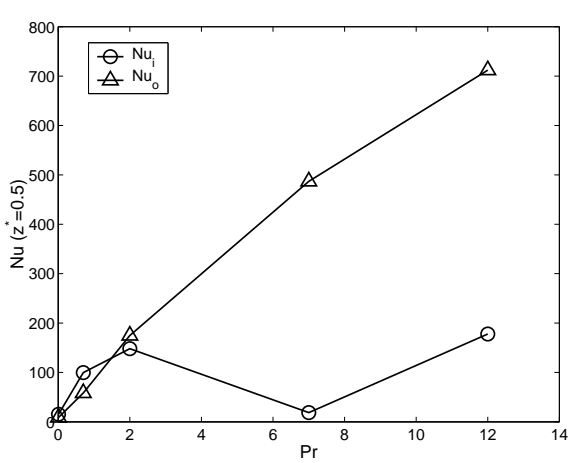

(b)

Figure 15: Local Nusselt numbers along both cylinders for $R e_{i}=10216, C_{w}=14858$ and different Prandtl numbers Pr: (a) Axial profiles; (b) Variations at mid-height $z^{*}=0.5$ against $\operatorname{Pr}$.

\section{Averaged Nusselt numbers}

From the isotherm maps (Fig.12) and using equations (3) and (4), we can calculate the averaged Nusselt numbers $\overline{N u_{i}}$ and $\overline{N u_{o}}$ for the inner and outer cylinders respectively. From an engineering point of view, it is quite interesting to provide correlations for $\overline{N u_{i}}$ and $\overline{N u_{o}}$ according to the flow control parameters $\left(R e_{i}, C_{w}\right.$ and $\operatorname{Pr}$ ). Figure 16 shows that the averaged Nusselt numbers on both cylinders can be correlated according to the three flow parameters as follows:

$$
\begin{aligned}
& \overline{N u_{i}}=0.0291 \times R e_{i}^{0.82} \times \operatorname{Pr}^{0.3} \times C_{w}^{0.09} \\
& \overline{N u_{o}}=0.0454 \times \operatorname{Re}_{i}^{0.75} \times \operatorname{Pr}^{0.8} \times C_{w}^{0.08}
\end{aligned}
$$

These two correlations are valid for $3744 \leq R e_{i} \leq 37443,0 \leq C_{w} \leq 30000$ and $0.01 \leq \operatorname{Pr} \leq 12$ for a cavity characterized by a small aspect ratio $L=0.013$ and a large radius ratio $s=0.961$ and for two given heating factors $\kappa_{i}=0.117$ and $\kappa_{o}=0.245$. 


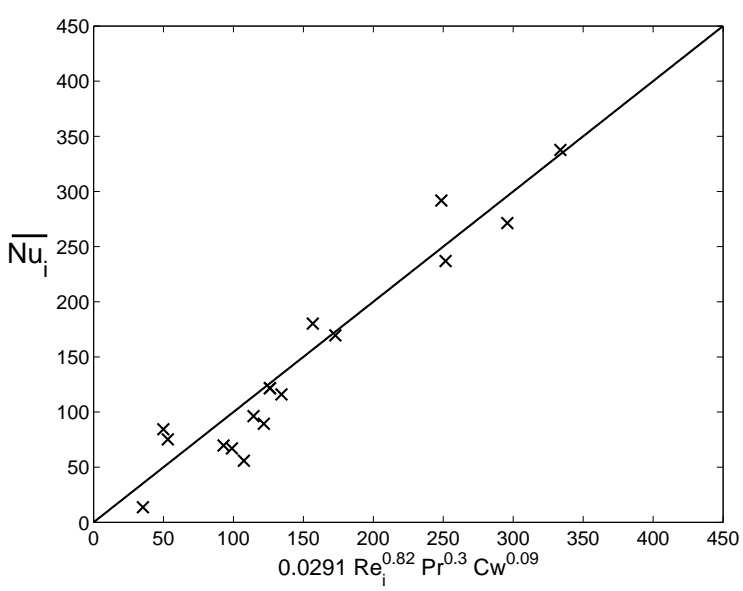

(a)

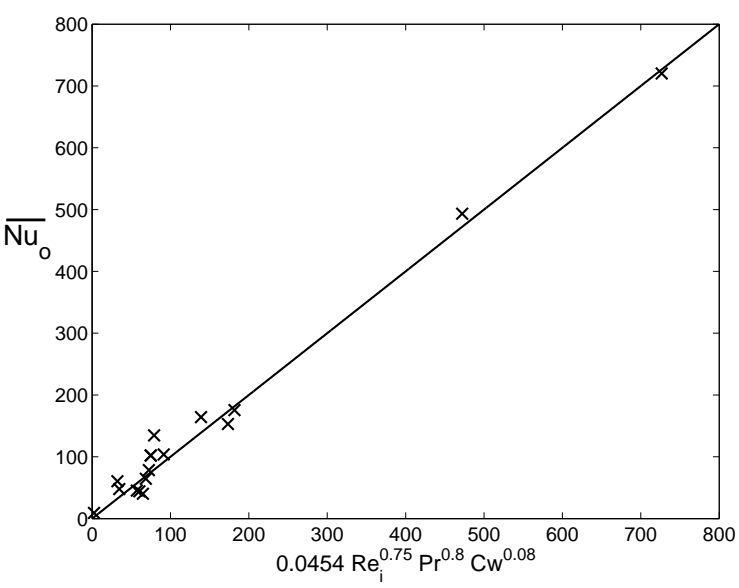

(b)

Figure 16: Correlations for the averaged Nusselt numbers along the: (a) inner cylinder and (b) the outer cylinder for all the considered cases.

These results are in agreement with previous results in various rotating flow arrangements. For turbulent rotating flows, the mean Nusselt number is usually proportional to $R e^{0.8}$ [38], which is the case in rotor-stator disk cavities [6] and also in Taylor-Couette systems [19]. In rotor-stator disk cavities, the exponent varies between 0.7 [39] and 0.89 [40], which confirms the relevance of the present results.

In the present case, we found that $\overline{N u_{i}} \propto C_{w}^{0.09}$ and $\overline{N u_{o}} \propto C_{w}^{0.08}$. To our knowledge, the only previous work providing a correlation with the flow rate coefficient is the one of Poncet and Schiestel [6] obtained using the same RSM model in the case of a rotor-stator interdisk cavity. These authors gave indeed the following correlation for the averaged Nusselt number along the stationary disk: $\overline{N u} \propto \operatorname{Re}^{0.8} \operatorname{Pr}^{0.5} C_{w}^{0.11}$ for a wide range of the parameters $5 \times 10^{5} \leq R e=\Omega R_{o}^{2} / \nu \leq 1.44 \times 10^{6}, 0 \leq C_{w} \leq 12082$ and $1 \leq \operatorname{Pr} \leq 12$. Our results appear then to be in good agreement with this previous study in an other rotating flow system.

The dependance of $\overline{N u_{i}}$ and $\overline{N u_{o}}$ on the Prandtl number is quite different between the two cylinders. In Taylor-Couette systems, $\overline{N u}$ is usually known to be proportional to $\operatorname{Pr}^{\beta}$, with $\beta$ equal or close to 0.3 , which is characteristic of heat transfer under the forced convection over a rotating cylinder. Aoki et al. [12] proposed $\beta=0.3$ for $360 \leq R e_{i} \leq 2274$ and Tachibana and Fukui [37] found $\beta=1 / 3$. Our results along the inner cylinder match quite well with these previous results as $\overline{N u_{i}} \propto \operatorname{Pr} r^{0.3}$ in the present case. But there is also a large variability in the values of $\beta$ depending especially on the temperature distributions imposed at the walls (effect of the Grashof number $G r$ or of the heating factors $\kappa_{i}$ and $\kappa_{o}$ ) and on the 
geometry (ratio $\Delta R / R_{i}$ ). Aoki et al. [12] showed indeed that $N u \propto G r^{0.29}$. Among others, we can cite the numerical work of Kuosa et al. [19] and the experimental one of Lee and Minkowycz [14], who proposed $\beta=0.4$ or the work of Simmers and Coney [41], who obtained $\overline{N u} \propto C(\operatorname{Pr}) \operatorname{Pr}$, with $C$ a function of $\operatorname{Pr}$. In the case of a turbulent rotor-stator flow, Owen and Haynes [42] found $\beta=0.6$. Thus, it appears difficult to provide definitive conclusions concerning the correlation between the averaged Nusselt number and the Prandt number along the outer cylinder. Nevertheless, the value $\beta=0.8$ found here, remains in the range $[0.4-1]$. The reader can refer to the $\mathrm{PhD}$ theses of Fasquelle [43] and Giret [9], who performed a large review of previous works in Taylor-Couette systems with or without axial throughflow, grooved or ungrooved cylinders and, who provided numerous correlations for the Nusselt number against all flow parameters.

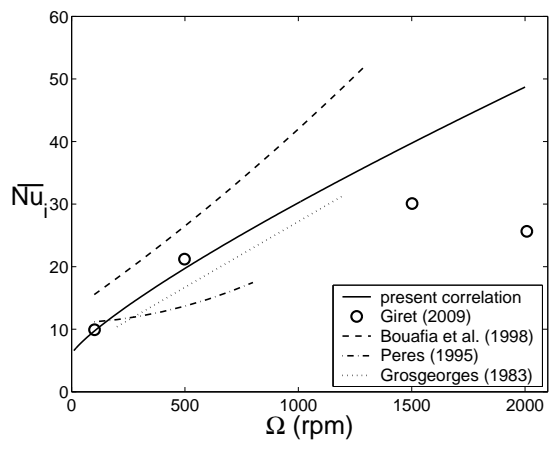

(a)

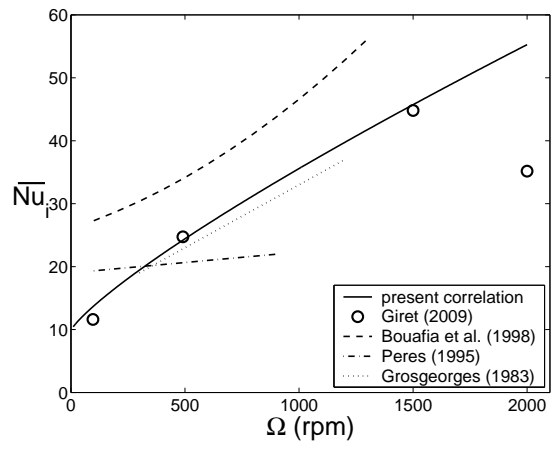

(b)

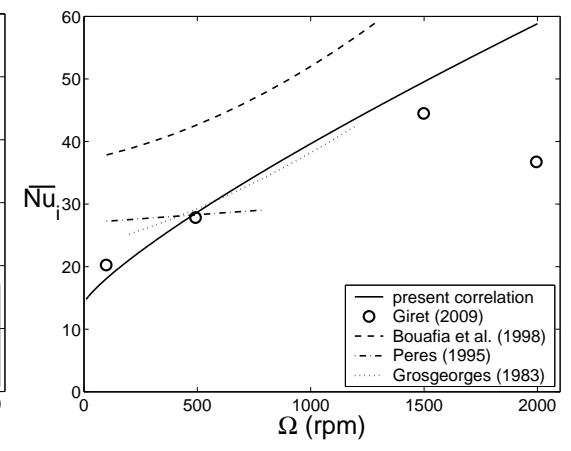

(c)

Figure 17: Avergaed Nusselt number along the rotor $\overline{N u_{i}}$ against the rotation rate $\Omega$ for $\operatorname{Pr}=0.7$ and three values of the flow rate coefficient: (a) $C_{w}=10684$ (mass flow rate of $30 \mathrm{~g} / \mathrm{s}$ ), (b) $C_{w}=21368$ (mass flow rate of 60 $\mathrm{g} / \mathrm{s}$ ), (c) $C_{w}=32052$ (mass flow rate of $90 \mathrm{~g} / \mathrm{s}$ ). Comparisons between the correlation (10) and previous results $[9,17,44,45]$.

To validate the correlation law (10) along the rotor and to highlight the influence of the rotation rate $\Omega$ and of the flow rate coefficient $C_{w}$ on the heat transfer coefficient, our results are compared to previous experimental $[9,17,44]$ and numerical [45] data for a given Prandtl number $\operatorname{Pr}=0.7$ and three values of the flow rate coefficient (see Figure 17). For these parameters, the law (10) reduces to the form:

$$
\overline{N u_{i}}=\overline{N u_{i 0}}+\alpha \times \Omega^{0.82}
$$

where the averaged Nusselt number $\overline{N u_{i 0}}$ has been obtained in the configuration of Giret [9] $(s=0.99$ and 
$L=0.003)$ without rotation $(\Omega=0)$. The coefficient $\alpha$ depends on $C_{w}^{0.09}$. The values of $\overline{N u_{i 0}}$ and $\alpha$ are given in Table IV for the three flow rates considered by Giret [9].

\begin{tabular}{c|c|c|c} 
mass flow rate $(\mathrm{g} / \mathrm{s})$ & $C_{w}$ & $\alpha$ & $\overline{N u_{i 0}}$ \\
\hline \hline 30 & 10684 & 0.533 & 6.03 \\
\hline 60 & 21368 & 0.568 & 9.85 \\
\hline 90 & 32052 & 0.589 & 14.17
\end{tabular}

Table IV: Coefficients involved in the empirical law (12) for the three flow rates considered by Giret [9].

A relatively good agreement is observed between the present results and the experimental data of Giret [9] for $\Omega$ up to $1500 \mathrm{rpm}$ whatever the value of $C_{w}$. The better agreement is obtained for the intermediate value of the flow rate coefficient $C_{w}=21368$. This new correlation (12) improves the correlation of Grosgeorges [44] and most of all the one of Bouafia et al. [17], which overestimates $\overline{N u_{i}}$ whatever the value of the rotation rate. The numerical results of Peres [45] fail to predict the good tendency as the averaged Nusselt number varies slightly against the rotation rate. For all correlations, the main discrepancy for the heat transfer coefficient along the rotor is observed for very high rotation rates $(\Omega=2000 \mathrm{rpm})$. Giret $[9]$ did not provide any explanation for the weak values of $\overline{N u_{i}}$ obtained for $\Omega=2000 \mathrm{rpm}$. Note that the same experimental results are not available along the stator, which prevents from doing the same comparisons along the other cylinder. 


\section{CONCLUSIONS}

In this study, the effects of the rotational speed, the flow rate and the working fluid on the hydrodynamic and thermal fields have been investigated numerically using the Reynolds Stress Modeling of Elena and Schiestel $[1,2]$ for the turbulent flow between two concentric cylinders where only the inner cylinder is rotating. Even if this model has been widely validated in various rotating cavities $[1,2,4,6]$, it has been also favorably compared to the velocity measurements of Escudier and Gouldson [7] for a turbulent flow in a very elongated Taylor-Couette system. In particular, it improves significantly the predictions of the $k-\varepsilon$ model of Naser [8] in the same configuration.

The RSM model has then be used in a Taylor-Couette-Poiseuille system defined by a radius ratio $s=0.961$ and an aspect ratio $L=0.013$ for a wide range of flow control parameters: rotational Reynolds number $R e_{i}$, flow rate coefficient $C_{w}$ and Prandtl number $\operatorname{Pr}$. The results showed that the flow is established quite rapidly. For axial positions larger than $20 \%$ of the cavity height, the radial profiles of the mean velocity components and also of the Reynolds stresses do not depend any more on the axial position. The mean flow is helical with a Poiseuille-like profile for the mean axial velocity component and a torsional Couette-like profile for the mean tangential velocity component. Turbulence is mainly concentrated in the middle of the gap between the two cylinders and vanishes towards the walls. The thermal field is also independent of the axial position for $z \geq 0.2 h$. The dominating influence of the outer cylinder on the thermal field has been shown. Finally, new correlations have been provided for the averaged Nusselt numbers along both cylinders according to the Reynolds and Prandtl numbers and to the flow rate coefficient and for a wide range of these parameters.

More comparisons with experimental data for the mean and turbulent hydrodynamic fields are now required before performing new calculations to investigate the effects of the geometry (aspect ratio $L$ and radius ratio $s)$ and of the heating factors $\left(\kappa_{i}\right.$ and $\left.\kappa_{o}\right)$ or the Grashof number on the heat transfer.

\section{ACKNOWLEDGEMENT}

The authors would thank Dr Roland Schiestel (CNRS, IRPHE) for fruitful discussions. This work was supported by Liebherr Aerospace Toulouse through a grant for Sofia Haddadi. 
[1] Elena, L. and Schiestel, R., 1996, "Turbulence modeling of rotating confined flows," Int. J. Heat and Fluid Flow, 17, pp. 283-289.

[2] Schiestel, R. and Elena, L., 1997, "Modeling of anisotropic turbulence in rapid rotation," Aerospace Science and Technology, 7, pp. 441-451.

[3] Poncet, S., 2005, Écoulements de type rotor-stator soumis à un flux axial: de Batchelor à Stewartson, Ph.D. thesis, Université de Provence.

[4] Poncet, S., Chauve, M. P., and Schiestel, R., 2005, "Batchelor versus Stewartson flow structures in a rotor-stator cavity with throughflow," Phys. Fluids, 17(075110).

[5] Haddadi, S. and Poncet, S., 2008, "Turbulence modeling of torsional couette flows," Int. J. Rotating Machinery, (Article ID 635138).

[6] Poncet, S. and Schiestel, R., 2007, "Numerical modeling of heat transfer and fluid flow in rotor-stator cavities with throughflow," Int. J. Heat Mass Transfer, 50(7-8), pp. 1528-1544.

[7] Escudier, M. P. and Gouldson, I. W., 1995, "Concentric annular flow with centerbody rotation of a newtonian and a shear-thinning liquid," Int. J. Heat and Fluid Flow, 16, pp. 156-162.

[8] Naser, J. A., 1997, "Prediction of newtonian and non-newtonian flow through concentric annulus with centerbody rotation," Int. Conf. on CFS in Mineral and Metal Processing and Power Generation, CSIRO.

[9] Giret, A., 2009, Transferts thermiques convectifs dans le cadre de machines tournantes, Ph.D. thesis, Université de Poitiers.

[10] Kaye, J. and Elgar, E. C., 1958, "Modes of adiabatic and diabatic fluid flow in an annulus with an inner rotating cylinder," Trans. ASME, 80, pp. 753-765.

[11] Becker, K. M. and Kaye, J., 1962, "Measurement of diabatic flow in an annulus with an inner rotating cylinder," Trans. ASME, Journal of Heat Transfer, 84, pp. 97-105.

[12] Aoki, H., Nohira, H., and Arai, H., 1967, "Convective heat transfer in an annulus with an inner rotating cylinder," Bulletin of JSME, 10(39), pp. 523-532.

[13] Kuzay, T. M. and Scott, C. J., 1977, "Turbulent heat transfer studies in annulus with inner cylinder rotation," J. Heat Transfer, 99, pp. 12-19.

[14] Lee, Y. N. and Minkowycz, W. J., 1989, "Heat transfer characteristics of the annulus of two-coaxial cylinders with one cylinder rotating," Int. J. Heat Mass Transfer, 32(4), pp. 711-722.

[15] Nouri, J. M. and Whitelaw, J. H., 1994, "Flow of newtonian and non-newtonian fluids in a concentric annulus 
with rotation of the inner cylinder," J. Fluid Eng., 116, pp. 821-827.

[16] Nouri, J. M. and Whitelaw, J. H., 1997, "Flow of newtonian and non-newtonian fluids in an eccentric annulus with rotation of the inner cylinder," Int. J. Heat Fluid Flow, 18, pp. 236-246.

[17] Bouafia, M., Bertin, Y., Saulnier, J., and Ropert, P., 1998, "Analyse expérimentale des transferts de chaleur en espace annulaire étroit et rainuré avec cylindre intérieur tournant," Int. J. Heat Mass Transfer, 41(10), pp. $1279-1291$.

[18] Char, M. I. and Hsu, Y. H., 1998, "Numerical prediction of turbulent mixed convection in a concentric horizontal rotating annulus with low-Re two-equation models," Int. J. Heat Mass Transfer, 41(12), pp. 1633-1643.

[19] Kuosa, M., Sallinen, P., and Larjola, J., 2004, "Numerical and experimental modelling of gas flow and heat transfer in the air gap of an electric machine," J. Thermal Science, 13(3), pp. 264-278.

[20] Chung, S. Y. and Sung, H. J., 2005, "Large-eddy simulation of turbulent flow in a concentric annulus with rotation of an inner cylinder," Int. J. Heat Fluid Flow, 26, pp. 191-203.

[21] Elena, L. and Schiestel, R., 1995, "Turbulence modeling of confined flow in rotating disk systems," AIAA J., 33(5), pp. 812-821.

[22] Poncet, S., Schiestel, R., and Monchaux, R., 2008, "Turbulence modeling of the Von Kármán flow: viscous and inertial stirrings," Int. J. Heat Fluid Flow, 29(1), pp. 62-74.

[23] Launder, B. E. and Tselepidakis, D. P., 1994, "Application of a new second-moment closure to turbulent channel flow rotating in orthogonal mode," Int. J. Heat and Fluid Flow, 15(1), pp. 2-10.

[24] Daly, B. J. and Harlow, F. H., 1970, “Transport equation for turbulence," Phys. Fluids A, 13(11), pp. $2634-2649$.

[25] Gibson, M. and Launder, B. E., 1978, "Ground effects on pressure fluctuations in the atmospheric boundary layer," J. Fluid. Mech., 86(3), pp. 491-511.

[26] Launder, B. E. and Reynolds, W. C., 1983, "Asymptotic near-wall stress dissipation rates in a turbulent flow," Phys. Fluids A, 26(5), pp. 1157-1158.

[27] Cambon, C. and Jacquin, L., 1989, "Spectral approach to non-isotropic turbulence subjected to rotation," J. Fluid. Mech., 202, pp. 295-317.

[28] Launder, B. E., Reynolds, W. C., Rodi, W., Mathieu, J., and Jeandel, D., 1984, Turbulence models and their applications, Ed. Eyrolles, Paris.

[29] Schiestel, R., 1998, Les écoulements turbulents: modélisation et simulation, Hermès, Paris.

[30] Ong, C. L., 1988, Computation of fluid flow and heat transfer in rotating disc systems, Ph.D. thesis, University of Sussex.

[31] Iacovides, H. and Chew, J. W., 1992, "The computation of convective heat transfer in rotating cavities," Int. J. 
Heat Fluid Flow, 14(2), pp. 146-154.

[32] Taylor, G. I., 1935, "Distribution of velocity and temperature between concentric rotating cylinders," Proc. Roy. Soc. A, 265, pp. 494-512.

[33] Polkowski, J. W., 1984, "Turbulent flow between coaxial cylinders with inner cylinder rotating," Trans. ASME, Journal for Gas Turbines and Power, 106(1), pp. 128-135.

[34] Lumley, J. L., 1978, "Computational modeling of turbulent flows," Advances in Applied Mechanics, 18, pp. 123-176.

[35] Hanjalic, K. and Launder, B. E., 1976, "Contribution towards a reynolds-stress closure for low-reynolds number turbulence," J. Fluid Mech., 74, pp. 583-610.

[36] Gazley, C., 1958, "Heat transfer characteristics of the rotational and axial flow between concentric cylinders," Transactions of the ASME, 80, pp. 79-90.

[37] Tachibana, F. and Fukui, S., 1964, "Convective heat transfer of the rotational and axial flow between two concentric cylinders," Bulletin of JSME, 7(26), pp. 385-391.

[38] Owen, J. M. and Rogers, R. H., 1989, Flow and Heat Transfer in Rotating-Disc Systems - Vol.1: Rotor-Stator Systems, Ed. Morris, W.D. John Wiley and Sons Inc., New-York.

[39] Poncet, S. and Serre, E., 2009, "High-order les of turbulent heat transfers in a rotor-stator cavity," Int. J. Heat Fluid Flow, 30(4), pp. 590-601.

[40] Roy, R. P., Xu, G., and Feng, J., 2001, "A study of convective heat transfer in a model rotor-stator disk cavity," ASME J. Turbomach., 123, pp. 621-632.

[41] Simmers, D. A. and Coney, J. E. R., 1979, "A Reynolds analogy solution for the heat transfer characteristics of combined Taylor vortex and axial flows," Int. J. Heat Mass Transfer, 22, pp. 679-689.

[42] Owen, J. M. and Haynes, C. M., 1976, "Design formulae for the heat loss and frictional resistance of air-cooled rotating discs - vol.4," Improvements in fluid mechanics and systems for energy conversion, Milan.

[43] Fasquelle, A., 2007, Contribution à la modélisation multi-physique: électro-vibro-acoustique et aérothermique de machines de traction, Ph.D. thesis, École Centrale de Lille.

[44] Grosgeorges, M., 1983, Contribution à l'étude du refroidisement d'une paroi tournante par air chargé d'huile, Ph.D. thesis, Université de Nancy.

[45] Peres, I., 1995, Contribution à l'analyse de l'écoulement et des transferts convectifs dans un espace annulaire lisse ou encoché par voie de simulations numériques. Application à un transfert de moteur électrique rapide, Ph.D. thesis, Université de Poitiers. 